\title{
The symmetric heavy-light ansatz
}

\author{
Dean Lee \\ Department of Physics, North Carolina State University, Raleigh, NC 27695
}

\begin{abstract}
The symmetric heavy-light ansatz is a method for finding the ground state of any dilute unpolarized system of attractive two-component fermions. Operationally it can be viewed as a generalization of the Kohn-Sham equations in density functional theory applied to $N$-body density correlations. While the original Hamiltonian has an exact $Z_{2}$ symmetry, the heavy-light ansatz breaks this symmetry by skewing the mass ratio of the two components. In the limit where one component is infinitely heavy, the many-body problem can be solved in terms of single-particle orbitals. The original $Z_{2}$ symmetry is recovered by enforcing $Z_{2}$ symmetry as a constraint on $N$-body density correlations for the two components. For the 1D, 2D, and 3D attractive Hubbard models the method is in very good agreement with exact Lanczos calculations for few-body systems at arbitrary coupling. For the 3D attractive Hubbard model there is very good agreement with lattice Monte Carlo results for many-body systems in the limit of infinite scattering length.
\end{abstract}




\section{INTRODUCTION}

Dilute two-component fermions with attractive interactions have some universal features of relevance to several areas of physics. In three dimensions the unpolarized ground state is believed to be superfluid with properties in between a Bardeen-Cooper-Schrieffer (BCS) fermionic superfluid at weak coupling and a Bose-Einstein condensate (BEC) of bound dimers at strong coupling [1, 2, 3]. The crossover transition occurs somewhere near the unitarity point, a scale-invariant point where the range of the interaction is zero and the scattering length is infinite. Much recent interest on this topic has been stimulated by experimental progress with cold atomic Fermi gases. Starting with a dilute Fermi gas, where the effective range of the interaction is negligible compared with the interparticle spacing, the scattering length can be tuned using a magnetic Feshbach resonance [4, 5, 6, , 7, 8, 9, 10]. In nuclear physics the phenomenology of the unitarity point is relevant to cold dilute neutron matter. The neutron scattering length is roughly $-18 \mathrm{fm}$ while the range of the interaction is comparable to the Compton wavelength of the pion, $m_{\pi}^{-1} \approx 1.4 \mathrm{fm}$. Therefore the unitarity point is approximately realized when the interparticle spacing is about $5 \mathrm{fm}$. This range of neutron density is expected in the inner crust of neutron stars.

In this paper we introduce a new theoretical method called the symmetric heavy-light ansatz. The ansatz is used to find the ground state energy of dilute unpolarized twocomponent fermions with attractive interactions. While our main interest is the threedimensional system, we demonstrate that the ansatz is accurate for any number of spatial dimensions. Operationally it can be described as an $N$-body generalization of the KohnSham equations in density functional theory. But rather than solving for one-body densities, we use the symmetric heavy-light ansatz to determine $N$-body density correlations. While useful as a numerical technique, the ansatz also provides a heuristic picture of the underlying competition between Fermi repulsion and attractive interactions.

We start with an effective Hamiltonian describing two-component fermions with shortrange attractive interactions. For the unpolarized case the ground state has an exact $Z_{2}$ symmetry corresponding with interchanging components. The first step of the ansatz is to break this $Z_{2}$ symmetry by skewing the mass ratio of the two components. In fact we take the extreme limit where one component is infinitely heavy. In this limit the many-body problem is completely solved in terms of single-particle orbitals. The original $Z_{2}$ symmetry 
is reintroduced as a constraint on the $N$-body correlations for the two components. In the simple approximation where only the lowest orbitals are filled, this constraint completely determines the $N$-body density correlations for each fermion component. We run the method through several numerical tests and find very good agreement for a number of exact Lanczos and Monte Carlo results for the attractive Hubbard model in one, two, and three dimensions. The low computational scaling of the ansatz allows us to make predictions for much larger systems which are not yet accessible using alternate methods.

The organization of the paper is as follows. The beginning is a short summary of symmetries of the effective Hamiltonian. After this we introduce the heavy-light Hamiltonian $H_{\mathrm{HL}}$ and the symmetric heavy-light ansatz. Some results relating to heavy-light orbitals are derived, and the spectrum of the heavy-light Hamiltonian $H_{\mathrm{HL}}$ is explored numerically for the unpolarized four-body system in three dimensions. This analysis provides motivation for the lowest filling approximation and the construction of $N$-body fixed-point densities. We then design a Markov chain algorithm to generate fixed-point densities. As a first test we compare the results of the symmetric heavy-light ansatz with exact Lanczos results for the four-body system in three dimensions. We also compare with exact results for fourand six-body systems in both one and two dimensions. We then consider larger systems in three dimensions at unitarity and the ground state energy for general values of the scattering length. We conclude with a discussion of some extensions of the method and future work.

\section{SYMMETRIES OF THE HAMILTONIAN}

In continuum notation we can write the effective Hamiltonian for two-component fermions in $d \leq 3$ dimensions with short-range interactions as

$$
H=-\frac{1}{2 m} \sum_{\sigma=\uparrow, \downarrow} \int d^{d} \vec{r} a_{\sigma}^{\dagger}(\vec{r}) \vec{\nabla}^{2} a_{\sigma}(\vec{r})+C \int d^{d} \vec{r} a_{\downarrow}^{\dagger}(\vec{r}) a_{\uparrow}^{\dagger}(\vec{r}) a_{\uparrow}(\vec{r}) a_{\downarrow}(\vec{r}) .
$$

Our main interest is the case $d=3$, but we also consider lower dimensional systems $d=1,2$. $a_{\sigma}$ and $a_{\sigma}^{\dagger}$ are annihilation and creation operators for fermions with two components. We refer to these components as up and down spins. The mass of the fermion is $m$, and the coefficient $C$ is assumed to be negative so that the interaction is attractive. The strength of $C$ depends on the scheme used to regulate the short distance behavior. This Hamiltonian 
has a global $U(1)$ fermion-number symmetry,

$$
\left[\begin{array}{l}
a_{\uparrow}(\vec{r}) \\
a_{\downarrow}(\vec{r})
\end{array}\right] \rightarrow e^{i \phi}\left[\begin{array}{l}
a_{\uparrow}(\vec{r}) \\
a_{\downarrow}(\vec{r})
\end{array}\right],
$$

where $\phi$ is any real constant. It also has a global $S U(2)$ spin symmetry

$$
\left[\begin{array}{l}
a_{\uparrow}(\vec{r}) \\
a_{\downarrow}(\vec{r})
\end{array}\right] \rightarrow e^{i \vec{\phi} \cdot \vec{\sigma}}\left[\begin{array}{l}
a_{\uparrow}(\vec{r}) \\
a_{\downarrow}(\vec{r})
\end{array}\right],
$$

where $\vec{\sigma}$ denotes the $2 \times 2$ Pauli spin matrices and $\vec{\phi}$ is any constant real three-component vector. Since there is no coupling between spin and orbital angular momentum, this $S U(2)$ symmetry should be regarded as an internal symmetry decoupled from spatial rotations.

The lowest-dimensional local bosonic operator that can be constructed from the annihilation field operators is

$$
\psi^{2}(\vec{r})=a_{\uparrow}(\vec{r}) a_{\downarrow}(\vec{r}) .
$$

We note that $\psi^{2}$ is invariant under the $S U(2)$ spin symmetry but phase rotates under the $U(1)$ fermion-number symmetry,

$$
\psi^{2}(\vec{r}) \rightarrow e^{2 i \phi} \psi^{2}(\vec{r})
$$

Therefore if there is some critical temperature below which $\psi^{2}$ has long-range spatial correlations,

$$
\lim _{|\vec{r}| \rightarrow \infty}\left\langle\psi^{2 \dagger}(\vec{r}) \psi^{2}(\overrightarrow{0})\right\rangle \neq 0
$$

then the $U(1)$ fermion-number symmetry is spontaneously broken. This condition of offdiagonal long-range order [11, 12] is the standard definition for superfluidity with S-wave pairing. We observe that the $S U(2)$ spin symmetry is not broken by the expectation value of $\psi^{2}$.

\section{SYMMETRIC HEAVY-LIGHT ANSATZ}

Let $K_{\uparrow}$ and $K_{\downarrow}$ be the kinetic energy operators associated with the up and down spins respectively,

$$
\begin{aligned}
& K_{\uparrow}=-\frac{1}{2 m} \int d^{d} \vec{r} a_{\uparrow}^{\dagger}(\vec{r}) \vec{\nabla}^{2} a_{\uparrow}(\vec{r}), \\
& K_{\downarrow}=-\frac{1}{2 m} \int d^{d} \vec{r} a_{\downarrow}^{\dagger}(\vec{r}) \vec{\nabla}^{2} a_{\downarrow}(\vec{r}) .
\end{aligned}
$$


We define $H_{\mathrm{HL}}$ as

$$
H_{\mathrm{HL}}=H-K_{\uparrow}+K_{\downarrow}=2 K_{\downarrow}+V,
$$

and refer to $H_{\mathrm{HL}}$ as the heavy-light Hamiltonian. In $H_{\mathrm{HL}}$ we have deleted the kinetic energy for the up spin while doubling the kinetic energy of the down spin. This could be viewed as introducing spin-dependent masses $m_{\uparrow}$ and $m_{\downarrow}$. For the original Hamiltonian $H$ we have $m_{\uparrow}=m_{\downarrow}=m$, while in $H_{\mathrm{HL}}$ we have $m_{\uparrow}=\infty, m_{\downarrow}=m / 2$.

The physics of the two-body system in the center-of-mass frame is exactly the same for $H$ and $H_{\mathrm{HL}}$. The reduced mass $\mu$ defined by

$$
\frac{1}{\mu}=\frac{1}{m_{\uparrow}}+\frac{1}{m_{\downarrow}}
$$

equals $m / 2$ in both cases. The exact equivalence of $H$ and $H_{\mathrm{HL}}$ for the center-of-mass twobody system is preserved by most regularization schemes such as dimensional regularization, momentum cutoff schemes, and Hamiltonian lattice regularization. Hamiltonian lattice regularization is discussed in the appendix.

While $H_{\mathrm{HL}}$ and $H$ are identical for the two-body system, they are very different for more than two particles. For example when the ultraviolet cutoff scale goes to infinity for the three-dimensional system, $H$ has a well-defined continuum limit while $H_{\mathrm{HL}}$ is unbounded below due to a clustering instability. The simplest example where this occurs is the threebody system consisting of two up spins and one down spin. It is known that this system collapses due to an attractive $1 / r^{2}$ potential when $m_{\uparrow} \gtrsim 13 m_{\downarrow}[13]$. On the other hand the clustering instability in $H_{\mathrm{HL}}$ is eliminated when we project onto quantum states invariant under the interchange of up and down spins. In the following we show that the minimum expectation value for $H_{\mathrm{HL}}$ restricted to this up-down symmetric space equals the ground state energy of $H$.

We denote a state with $N_{\uparrow}$ up spins and $N_{\downarrow}$ down spins as an $N_{\uparrow}, N_{\downarrow}$ state. We also specify the total momentum $\vec{P}$ and total spin $S$ of the $S U(2)$ spin representation. Let $\left|\Psi_{N, N}^{0}\right\rangle$ be the normalized ground state of $H$ for the $N, N$ system in a periodic cube of length $L$. Since the $S U(2)$ spin symmetry is not broken by the expectation value of $\psi^{2}$, we assume that $\left|\Psi_{N, N}^{0}\right\rangle$ lies in the spin-invariant sector $S=0$. Let $E_{N, N}^{0}$ be the corresponding ground state energy. Let $U$ be any unitary operator which interchanges the up and down 
spins through a $\pi$-radian spin rotation. Without loss of generality we take $U$ to be

$$
U=\exp \left[-i \frac{\pi}{2} \int d^{d} \vec{r} a_{\downarrow}^{\dagger}(\vec{r}) a_{\uparrow}(\vec{r})-i \frac{\pi}{2} \int d^{d} \vec{r} a_{\uparrow}^{\dagger}(\vec{r}) a_{\downarrow}(\vec{r})\right] .
$$

Clearly

$$
\begin{aligned}
& U^{\dagger} a_{\uparrow} U=-i a_{\downarrow}, \\
& U^{\dagger} a_{\downarrow} U=-i a_{\uparrow} .
\end{aligned}
$$

The phases appearing in Eq. (12) and (13) are necessary in order that the pair annihilation operator $\psi^{2}(\vec{r})$ remains invariant,

$$
U^{\dagger} \psi^{2}(\vec{r}) U=U^{\dagger} a_{\uparrow}(\vec{r}) a_{\downarrow}(\vec{r}) U=-a_{\downarrow}(\vec{r}) a_{\uparrow}(\vec{r})=\psi^{2}(\vec{r}) .
$$

Any state with an even number of fermions is invariant under two successive transformations of $U$. Therefore $U$ acting on the space of even fermion states generates a unitary representation of the group $Z_{2}$.

Let $\left|\Phi_{N, N}\right\rangle$ be any $N, N$ state which is $Z_{2}$ invariant,

$$
U\left|\Phi_{N, N}\right\rangle=\left|\Phi_{N, N}\right\rangle
$$

Since

$$
\left\langle\Phi_{N, N}\left|K_{\uparrow}\right| \Phi_{N, N}\right\rangle=\left\langle\Phi_{N, N}\left|K_{\downarrow}\right| \Phi_{N, N}\right\rangle
$$

it follows that

$$
\left\langle\Phi_{N, N}\left|H_{\mathrm{HL}}\right| \Phi_{N, N}\right\rangle=\left\langle\Phi_{N, N}|H| \Phi_{N, N}\right\rangle
$$

In particular this means

$$
\min _{U\left|\Phi_{N, N}\right\rangle=\left|\Phi_{N, N}\right\rangle} \frac{\left\langle\Phi_{N, N}\left|H_{\mathrm{HL}}\right| \Phi_{N, N}\right\rangle}{\left\langle\Phi_{N, N} \mid \Phi_{N, N}\right\rangle}=\min _{U\left|\Phi_{N, N}\right\rangle=\left|\Phi_{N, N}\right\rangle} \frac{\left\langle\Phi_{N, N}|H| \Phi_{N, N}\right\rangle}{\left\langle\Phi_{N, N} \mid \Phi_{N, N}\right\rangle} .
$$

Since $\left|\Psi_{N, N}^{0}\right\rangle$ is a $Z_{2}$-invariant state we conclude that

$$
\min _{U\left|\Phi_{N, N}\right\rangle=\left|\Phi_{N, N}\right\rangle} \frac{\left\langle\Phi_{N, N}\left|H_{\mathrm{HL}}\right| \Phi_{N, N}\right\rangle}{\left\langle\Phi_{N, N} \mid \Phi_{N, N}\right\rangle}=E_{N, N}^{0} .
$$

We refer to this exact relation as the symmetric heavy-light ansatz. The task of computing the ground state energy $E_{N, N}^{0}$ is reduced to finding the minimum of value of the Rayleigh-Ritz ratio

$$
\frac{\left\langle\Phi_{N, N}\left|H_{\mathrm{HL}}\right| \Phi_{N, N}\right\rangle}{\left\langle\Phi_{N, N} \mid \Phi_{N, N}\right\rangle}
$$

under the constraint of $Z_{2}$ invariance,

$$
U\left|\Phi_{N, N}\right\rangle=\left|\Phi_{N, N}\right\rangle
$$




\section{HEAVY-LIGHT ORBITALS}

In this section we discuss some properties of the energy eigenstates of the heavy-light Hamiltonian $H_{\mathrm{HL}}$. Since the up spins are infinitely massive, we can fix these particles at

locations $\vec{R}_{1}, \vec{R}_{2}, \cdots, \vec{R}_{N}$. Due to antisymmetry each $\vec{R}_{i}$ must be distinct, and we use the shorthand notation

$$
\mathbf{R}=\left\{\vec{R}_{1}, \vec{R}_{2}, \cdots, \vec{R}_{N}\right\}
$$

for the unordered set of vectors. We define $|\mathbf{R}\rangle$ as the corresponding antisymmetric state of localized particles,

$$
|\mathbf{R}\rangle=\frac{1}{\sqrt{N !}} \sum_{\pi} \operatorname{sgn}(\pi)\left|\vec{R}_{\pi(1)}\right\rangle \otimes\left|\vec{R}_{\pi(2)}\right\rangle \otimes \cdots\left|\vec{R}_{\pi(N)}\right\rangle .
$$

The summation is over all permutations $\pi$ of the integers $1,2, \cdots, N$. $\operatorname{sgn}(\pi)$ equals -1 for odd permutations and +1 for even permutations. The normalization for $|\mathbf{R}\rangle$ is then simply

$$
\left\langle\mathbf{R}^{\prime} \mid \mathbf{R}\right\rangle=\operatorname{det} \Delta_{i j}\left(\mathbf{R}^{\prime}, \mathbf{R}\right)
$$

where

$$
\Delta_{i j}\left(\mathbf{R}^{\prime}, \mathbf{R}\right)=\delta^{(d)}\left(\vec{R}_{i}^{\prime}-\vec{R}_{j}\right) .
$$

Later in the discussion when we consider lattice models the Dirac delta functions will be replaced by Kronecker delta functions.

For any given $\mathbf{R}$ the down spins see a static delta function potential from each up spin,

$$
\sum_{i=1, \cdots, N} C \delta^{(d)}\left(\vec{r}-\vec{R}_{i}\right)
$$

For the very special case where $\mathbf{R}$ is a regular cubic array, this system is known as the Kronig-Penney model [14]. In general though $\mathbf{R}$ is irregular without translational and rotational symmetries. For a given $\mathbf{R}$ let the normalized single-particle orbitals be $\left|f_{j}(\mathbf{R})\right\rangle$ with corresponding eigenvalues $E_{j}(\mathbf{R})$. By convention we label the orbitals so that $E_{j}(\mathbf{R})$ increases with $j$,

$$
E_{1}(\mathbf{R}) \leq E_{2}(\mathbf{R}) \leq \cdots \leq E_{j}(\mathbf{R}) \leq \cdots
$$

For a single orbital we write the position-space wavefunction as

$$
f_{j}(\vec{r}, \mathbf{R})=\left\langle\vec{r} \mid f_{j}(\mathbf{R})\right\rangle .
$$


Analogous to $\mathbf{R}$, we define

$$
\mathbf{r}=\left\{\vec{r}_{1}, \vec{r}_{2}, \cdots, \vec{r}_{N}\right\}
$$

for the unordered set of distinct vectors $\vec{r}_{i}$. These correspond with possible locations for the $N$ down spins. We also define the antisymmetric product of position eigenstates

$$
|\mathbf{r}\rangle=\frac{1}{\sqrt{N !}} \sum_{\pi} \operatorname{sgn}(\pi)\left|\vec{r}_{\pi(1)}\right\rangle \otimes\left|\vec{r}_{\pi(2)}\right\rangle \otimes \cdots\left|\vec{r}_{\pi(N)}\right\rangle
$$

with normalization

$$
\left\langle\mathbf{r}^{\prime} \mid \mathbf{r}\right\rangle=\operatorname{det} \Delta_{i j}\left(\mathbf{r}^{\prime}, \mathbf{r}\right)
$$

Since there is no interaction between down spins, each eigenstate of $H_{\mathrm{HL}}$ for fixed $\mathbf{R}$ is an antisymmetric product of single-particle orbitals. Let us write the normalized quantum state with $N$ down spins filling orbitals $j_{1}, \cdots, j_{N}$ as

$$
\bigwedge_{n=1, \cdots, N}\left|f_{j_{n}}(\mathbf{R})\right\rangle=\frac{1}{\sqrt{N !}} \sum_{\pi} \operatorname{sgn}(\pi)\left|f_{\pi\left(j_{1}\right)}(\mathbf{R})\right\rangle \otimes\left|f_{\pi\left(j_{2}\right)}(\mathbf{R})\right\rangle \otimes \cdots\left|f_{\pi\left(j_{N}\right)}(\mathbf{R})\right\rangle .
$$

The position state wavefunction for this state is a Slater determinant,

$$
\left\langle\mathbf{r}\left|\bigwedge_{n=1, \cdots, N}\right| f_{j_{n}}(\mathbf{R})\right\rangle=\operatorname{det}\left[\begin{array}{cccc}
f_{j_{1}}\left(\vec{r}_{1}, \mathbf{R}\right) & f_{j_{2}}\left(\vec{r}_{1}, \mathbf{R}\right) & \cdots & f_{j_{N}}\left(\vec{r}_{1}, \mathbf{R}\right) \\
f_{j_{1}}\left(\vec{r}_{2}, \mathbf{R}\right) & f_{j_{2}}\left(\vec{r}_{2}, \mathbf{R}\right) & \cdots & f_{j_{N}}\left(\vec{r}_{2}, \mathbf{R}\right) \\
\vdots & \vdots & \ddots & \vdots \\
f_{j_{1}}\left(\vec{r}_{N}, \mathbf{R}\right) & f_{j_{2}}\left(\vec{r}_{N}, \mathbf{R}\right) & \cdots & f_{j_{N}}\left(\vec{r}_{1}, \mathbf{R}\right)
\end{array}\right]
$$

All eigenstates of $H_{\mathrm{HL}}$ are a tensor product of up-spin position eigenstates and down-spin orbitals,

$$
|\mathbf{R}\rangle \otimes \bigwedge_{n=1, \cdots, N}\left|f_{j_{n}}(\mathbf{R})\right\rangle
$$

with corresponding eigenvalue

$$
\sum_{n=1, \cdots, N} E_{j_{n}}(\mathbf{R})
$$

We can use the eigenstates of $H_{\mathrm{HL}}$ to perform a basis decomposition of the ground state $\left|\Psi_{N, N}^{0}\right\rangle$. For any given $\mathbf{R}$ and orbital indices $j_{1}, \cdots, j_{N}$, we write the inner product with the ground state as

$$
F_{\mathbf{R},\left\{j_{n}\right\}}=\left[\left\langle\mathbf{R}\left|\otimes \bigwedge_{n=1, \cdots, N}\left\langle f_{j_{n}}(\mathbf{R})\right|\right] \mid \Psi_{N, N}^{0}\right\rangle\right.
$$


with normalization

$$
\int d^{d N} \mathbf{R} \sum_{\left\{j_{n}\right\}}\left|F_{\mathbf{R},\left\{j_{n}\right\}}\right|^{2}=1
$$

We find that

$$
\begin{aligned}
E_{N, N}^{0} & =\left\langle\Psi_{N, N}^{0}|H| \Psi_{N, N}^{0}\right\rangle=\left\langle\Psi_{N, N}^{0}\left|H_{\mathrm{HL}}\right| \Psi_{N, N}^{0}\right\rangle \\
& =\int d^{d N} \mathbf{R} \sum_{\left\{j_{n}\right\}}\left|F_{\mathbf{R},\left\{j_{n}\right\}}\right|^{2} \sum_{n=1, \cdots, N} E_{j_{n}}(\mathbf{R}) .
\end{aligned}
$$

The coefficients $\left|F_{\mathbf{R},\left\{j_{n}\right\}}\right|^{2}$ define a normalized probability distribution. Weighted by this probability distribution, $E_{N, N}^{0}$ is the average over all $\mathbf{R}$ and $\left\{j_{n}\right\}$ of the orbital energy sums

$$
\sum_{n=1, \cdots, N} E_{j_{n}}(\mathbf{R})
$$

The energy constraint Eq. (38) is an exact relation satisfied by the ground state energy $E_{N, N}^{0}$ of the original Hamiltonian $H$. Since the phase of $F_{\mathbf{R},\left\{j_{n}\right\}}$ is irrelevant, solving this constraint is qualitatively easier than solving for the ground state wavefunction $\left|\Psi_{N, N}^{0}\right\rangle$. In this respect Eq. (38) can be regarded as the starting point for a generalized density functional approach. However instead of single-particle densities we work with many-body densities given by the coefficients $\left|F_{\mathbf{R},\left\{j_{n}\right\}}\right|^{2}$.

\section{V. $\quad H$ AND $H_{\text {HL }}$ ON THE LATTICE}

Throughout this section we discuss Hamiltonian lattice regularization for interacting twocomponent fermions with short-range interactions. Further details for both Hamiltonian and Euclidean lattice formulations can be found in [15, 16, 17, 18, 19, 20, 21, 22, 23, 24, 25, 26, 27].

\section{A. Attractive Hubbard model}

On a $d$-dimensional spatial lattice we can write $H$ as

$$
H=K_{\uparrow}+K_{\downarrow}+V
$$

where

$$
K_{\uparrow}=\frac{1}{2 m} \sum_{\vec{r}, l}\left[2 a_{\uparrow}^{\dagger}(\vec{r}) a_{\uparrow}(\vec{r})-a_{\uparrow}^{\dagger}(\vec{r}) a_{\uparrow}(\vec{r}+\hat{l})-a_{\uparrow}^{\dagger}(\vec{r}) a_{\uparrow}(\vec{r}-\hat{l})\right],
$$




$$
K_{\downarrow}=\frac{1}{2 m} \sum_{\vec{r}, l}\left[2 a_{\downarrow}^{\dagger}(\vec{r}) a_{\downarrow}(\vec{r})-a_{\downarrow}^{\dagger}(\vec{r}) a_{\downarrow}(\vec{r}+\hat{l})-a_{\downarrow}^{\dagger}(\vec{r}) a_{\downarrow}(\vec{r}-\hat{l})\right],
$$

and

$$
V=C \sum_{\vec{r}} a_{\downarrow}^{\dagger}(\vec{r}) a_{\uparrow}^{\dagger}(\vec{r}) a_{\uparrow}(\vec{r}) a_{\downarrow}(\vec{r})
$$

Here $\vec{r}$ is an integer-valued $d$-dimensional spatial lattice vector, and $\hat{l}=\hat{1}, \ldots, \hat{d}$ are lattice unit vectors in each of the spatial directions. We write $m$ for the fermion mass and $C$ for the coupling constant. Throughout we use dimensionless parameters and operators, which correspond with physical values multiplied by the appropriate power of the spatial lattice spacing $a$. This lattice model is the same as the $d$-dimensional attractive Hubbard model. The Hamiltonian for the Hubbard model is usually written as

$$
H_{\text {Hubbard }}=-t \sum_{\vec{r}, l, \sigma=\uparrow, \downarrow}\left[a_{\sigma}^{\dagger}(\vec{r}) a_{\sigma}(\vec{r}+\hat{l})+a_{\sigma}^{\dagger}(\vec{r}) a_{\sigma}(\vec{r}-\hat{l})\right]+U \sum_{\vec{r}} a_{\downarrow}^{\dagger}(\vec{r}) a_{\uparrow}^{\dagger}(\vec{r}) a_{\uparrow}(\vec{r}) a_{\downarrow}(\vec{r}) .
$$

Therefore in $d$ dimensions,

$$
H_{\text {Hubbard }}=H+\left(N_{\uparrow}+N_{\downarrow}\right) \frac{d}{m}
$$

and

$$
U=C, \quad t=\frac{1}{2 m} .
$$

We define the heavy-light Hamiltonian on the lattice as

$$
H_{\mathrm{HL}}=H-K_{\uparrow}+K_{\downarrow}=2 K_{\downarrow}+V
$$

As in the continuum case the change from $H$ to $H_{\mathrm{HL}}$ can be viewed as altering the masses of the spins. In $H$ we have $m_{\uparrow}=m_{\downarrow}=m$, while in $H_{\mathrm{HL}}$ we have $m_{\uparrow}=\infty, m_{\downarrow}=m / 2$. More generally we can define

$$
H(\theta)=H+\left(-K_{\uparrow}+K_{\downarrow}\right) \theta=(1-\theta) K_{\uparrow}+(1+\theta) K_{\downarrow}+V
$$

which interpolates between $H$ at $\theta=0$ and $H_{\mathrm{HL}}$ at $\theta=1$. In terms of the spin-dependent masses $m_{\uparrow}$ and $m_{\downarrow}$,

$$
\theta=\frac{m_{\uparrow}-m_{\downarrow}}{m_{\uparrow}+m_{\downarrow}} .
$$




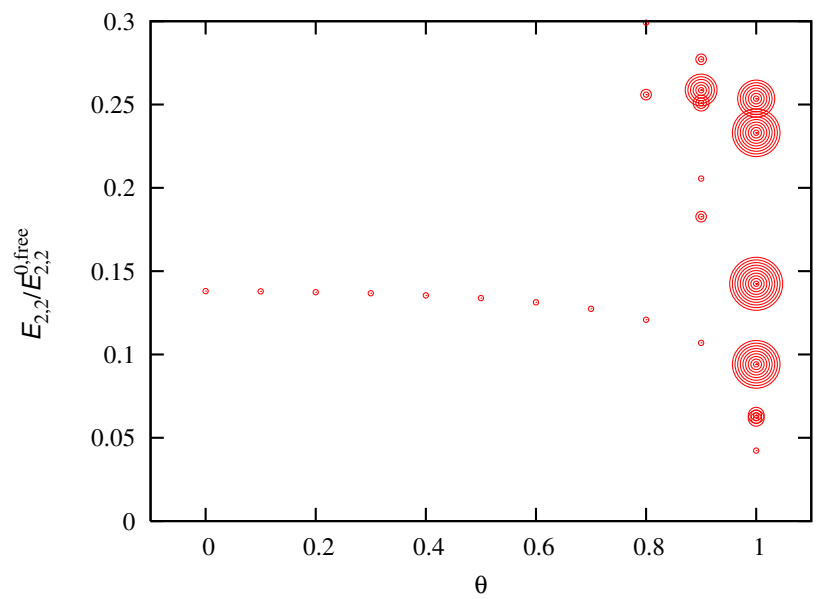

FIG. 1: Low-energy spectrum of $H(\theta)$ at total momentum $\vec{P}=0$ for the four-body system on

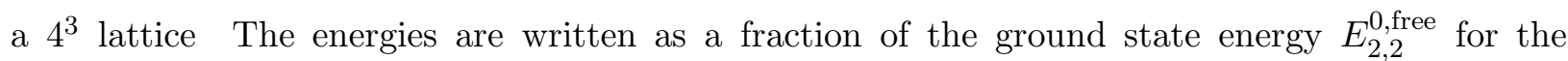
non-interacting system. The coupling constant $C$ is tuned to the unitarity point.

\section{B. Spectrum of the four-body system in three dimensions}

To gain some intuition for the low-energy spectrum of $H(\theta)$, we consider the unpolarized four-body system, $N_{\uparrow}=N_{\downarrow}=2$, in three dimensions. We tune the coupling constant $C$ to the unitarity limit where the scattering length is infinite. This procedure uses Lüscher's finite-volume scattering formula and is discussed in the appendix. In Fig. 1 we show the low-energy spectrum of $H(\theta)$ for the four-body system at total momentum $\vec{P}=0$ on a $4^{3}$ lattice. The energies are computed using the Lanczos method [28] and written as a

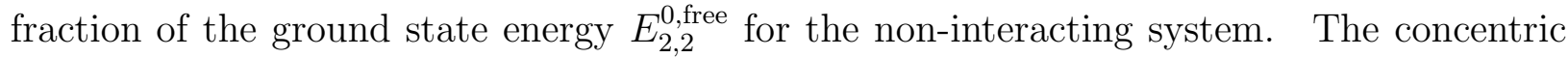
circles indicate exact degeneracies as well as approximate degeneracies too close to visually distinguish in the plot.

The ground state energy remains relatively constant for $\theta<0.6$. For larger $\theta$ the ground state energy decreases more substantially while the density of low-energy states increases. The data at $\theta=1$ corresponds with the spectrum of the heavy-light Hamiltonian $H_{\mathrm{HL}}$. From

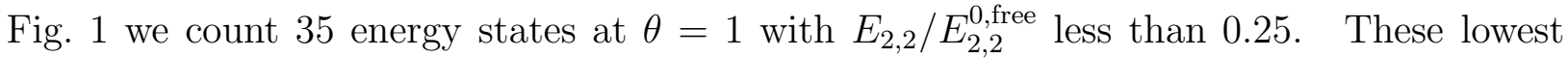
35 energy states correspond with the 35 independent states obtained by placing down spins in the lowest two energy orbitals for each possible up-spin configuration $\mathbf{R}=\left\{\vec{R}_{1}, \vec{R}_{2}\right\}$, and then projecting onto $\vec{P}=0$. A sketch of the one-body density distribution of down spins 


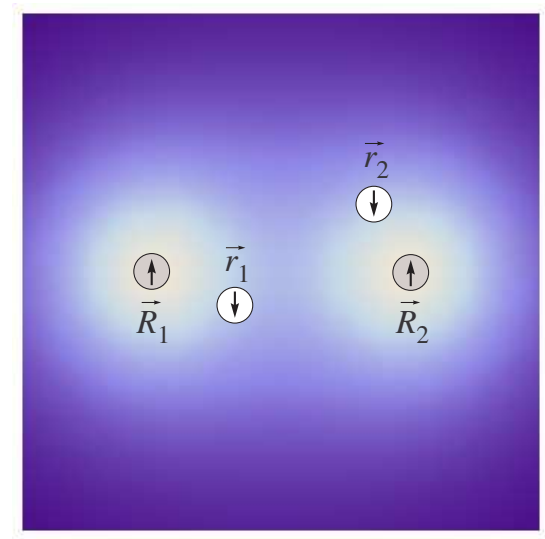

FIG. 2: Sketch of the one-body density distribution of down spins in the lowest orbitals for a fixed configuration of up spins.

in the lowest orbitals for a fixed configuration of up spins is shown in Fig. 2 ,

\section{LOWEST FILLING APPROXIMATION}

\section{A. Lowest orbital filling and effective distribution $\left|F_{\mathbf{R}}\right|^{2}$}

On the lattice we write a discrete sum over $\mathbf{R}$ rather than a continuous integral. Therefore Eq. (38) becomes

$$
E_{N, N}^{0}=\sum_{\mathbf{R}} \sum_{\left\{j_{n}\right\}}\left|F_{\mathbf{R},\left\{j_{n}\right\}}\right|^{2} \sum_{n=1, \cdots, N} E_{j_{n}}(\mathbf{R})
$$

In our example of the four-body system in three dimensions at unitarity, we found that for each $\mathbf{R}$ the sum

$$
\sum_{n=1,2} E_{j_{n}}(\mathbf{R})
$$

is numerically close to $E_{2,2}^{0}$ only when the down spins occupy the lowest orbitals, $\left\{j_{n}\right\}=$ $\{1,2\}$. Given that the weighted average

$$
\sum_{\mathbf{R}} \sum_{\left\{j_{n}\right\}}\left|F_{\mathbf{R},\left\{j_{n}\right\}}\right|^{2} \sum_{n=1,2} E_{j_{n}}(\mathbf{R})
$$

equals $E_{2,2}^{0}$, we conclude that $\left|F_{\mathbf{R},\left\{j_{n}\right\}}\right|^{2}$ is dominated by the set of lowest orbitals $\left\{j_{n}\right\}=$ $\{1,2\}$ for each $\mathbf{R}$. This suggests a general approximation scheme which keeps only the lowest orbitals. 
As in our four-body example at unitarity, let us assume that most of the weight of the normalized distribution $\left|F_{\mathbf{R},\left\{j_{n}\right\}}\right|^{2}$ lies in orbital sets $\left\{j_{n}\right\}$ where all orbitals lie near the bottom $N$ in energy. Let

$$
F_{\mathbf{R}}=\sqrt{\sum_{\left\{j_{n}\right\}}\left|F_{\mathbf{R},\left\{j_{n}\right\}}\right|^{2}}
$$

Then

$$
E_{N, N}^{0}=\sum_{\mathbf{R}} \sum_{\left\{j_{n}\right\}}\left|F_{\mathbf{R},\left\{j_{n}\right\}}\right|^{2} \sum_{n=1, \cdots, N} E_{j_{n}}(\mathbf{R}) \approx \sum_{\mathbf{R}}\left|F_{\mathbf{R}}\right|^{2} \sum_{j=1, \cdots, N} E_{j}(\mathbf{R}) .
$$

This approximation uses an effective distribution with weight $\left|F_{\mathbf{R}}\right|^{2}$ which is nonzero only for $\left\{j_{n}\right\}=\{1, \cdots, N\}$. We refer to this as the lowest filling approximation.

\section{B. N-body fixed-point densities}

In the lowest filling approximation we are restricted to states of the general form

$$
\begin{gathered}
\left|\Phi_{N, N}\right\rangle=\sum_{\mathbf{R}} F_{\mathbf{R}}\left[|\mathbf{R}\rangle \otimes \bigwedge_{j=1, \cdots, N}\left|f_{j}(\mathbf{R})\right\rangle\right], \\
\sum_{\mathbf{R}}\left|F_{\mathbf{R}}\right|^{2}=1 .
\end{gathered}
$$

In general any $\left|\Phi_{N, N}\right\rangle$ of this form will not satisfy the $Z_{2}$-invariance condition $U\left|\Phi_{N, N}\right\rangle=$ $\left|\Phi_{N, N}\right\rangle$ exactly. Therefore we search for a weaker symmetry constraint that follows from $Z_{2}$ invariance but can be satisfied exactly in the lowest filling approximation. In the following we design a constraint which is exactly solvable in the lowest filling approximation, with a unique solution $\left|F_{\mathbf{R}}\right|^{2}$ for each $\mathbf{R}$.

Let us define the up-spin and down-spin particle densities,

$$
\begin{aligned}
& \rho_{\uparrow}(\vec{r})=a_{\uparrow}^{\dagger}(\vec{r}) a_{\uparrow}(\vec{r}), \\
& \rho_{\downarrow}(\vec{r})=a_{\downarrow}^{\dagger}(\vec{r}) a_{\downarrow}(\vec{r}) .
\end{aligned}
$$

Let $G\left[\rho_{\uparrow}, \rho_{\downarrow}\right]$ be any functional involving the particle densities. Then any state $\left|\Phi_{N, N}\right\rangle$ satisfying the invariance condition $U\left|\Phi_{N, N}\right\rangle=\left|\Phi_{N, N}\right\rangle$ also satisfies

$$
\left\langle\Phi_{N, N}\left|: G\left[\rho_{\uparrow}, \rho_{\downarrow}\right]:\right| \Phi_{N, N}\right\rangle=\left\langle\Phi_{N, N}\left|: G\left[\rho_{\downarrow}, \rho_{\uparrow}\right]:\right| \Phi_{N, N}\right\rangle .
$$

In particular we have

$$
\left\langle\Phi_{N, N}\left|: \rho_{\uparrow}\left(\vec{r}_{1}\right) \times \cdots \times \rho_{\uparrow}\left(\vec{r}_{N}\right):\right| \Phi_{N, N}\right\rangle=\left\langle\Phi_{N, N}\left|: \rho_{\downarrow}\left(\vec{r}_{1}\right) \times \cdots \times \rho_{\downarrow}\left(\vec{r}_{N}\right):\right| \Phi_{N, N}\right\rangle .
$$


The : symbols denote normal ordering. Upon summation this constraint implies analogous relations for the $j$-body densities for each $j<N$,

$$
\left\langle\Phi_{N, N}\left|: \rho_{\uparrow}\left(\vec{r}_{1}\right) \times \cdots \times \rho_{\uparrow}\left(\vec{r}_{j}\right):\right| \Phi_{N, N}\right\rangle=\left\langle\Phi_{N, N}\left|: \rho_{\downarrow}\left(\vec{r}_{1}\right) \times \cdots \times \rho_{\downarrow}\left(\vec{r}_{j}\right):\right| \Phi_{N, N}\right\rangle .
$$

We can write the $N$-body up-spin densities in terms of $F_{\mathbf{R}}$,

$$
\begin{aligned}
\left\langle\Phi_{N, N}\right| & : \rho_{\uparrow}\left(\vec{R}_{1}\right) \times \cdots \times \rho_{\uparrow}\left(\vec{R}_{N}\right):\left|\Phi_{N, N}\right\rangle \\
& =\left\langle\Phi_{N, N}\left|a_{\uparrow}^{\dagger}\left(\vec{R}_{N}\right) \times \cdots \times a_{\uparrow}^{\dagger}\left(\vec{R}_{1}\right) a_{\uparrow}\left(\vec{R}_{1}\right) \times \cdots \times a_{\uparrow}\left(\vec{R}_{N}\right)\right| \Phi_{N, N}\right\rangle=N !\left|F_{\mathbf{R}}\right|^{2} .
\end{aligned}
$$

We can write the $N$-body down-spin densities in terms of $F_{\mathbf{R}}$ and the inner product $\left\langle\mathbf{r}\left|\bigwedge_{j=1, \cdots, N}\right| f_{j}(\mathbf{R})\right\rangle$

$$
\left\langle\Phi_{N, N}\left|: \rho_{\downarrow}\left(\vec{r}_{1}\right) \times \cdots \times \rho_{\downarrow}\left(\vec{r}_{N}\right):\right| \Phi_{N, N}\right\rangle=N ! \sum_{\mathbf{R}}\left|\left\langle\mathbf{r}\left|\bigwedge_{j=1, \cdots, N}\right| f_{j}(\mathbf{R})\right\rangle\right|^{2} \times\left|F_{\mathbf{R}}\right|^{2} .
$$

If the $N$-body densities for up spins and down spins are equal then

$$
\sum_{\mathbf{R}}\left|\left\langle\mathbf{r}\left|\bigwedge_{j=1, \cdots, N}\right| f_{j}(\mathbf{R})\right\rangle\right|^{2} \times\left|F_{\mathbf{R}}\right|^{2}=\left|F_{\mathbf{r}}\right|^{2}
$$

We refer to any $\left|F_{\mathbf{R}}\right|^{2}$ which satisfies this relation an $N$-body fixed-point density. The existence and uniqueness of fixed-point solutions $\left|F_{\mathbf{R}}\right|^{2}$ will be proved in the next section.

At this point we comment on the clustering instability of $H_{\mathrm{HL}}$. In two dimensions when any two of the up-spin locations $\vec{R}_{i}$ and $\vec{R}_{j}$ come close together, the lowest heavy-light orbital energy in physical units has a divergence proportional to $a^{-1}$, where $a$ is the lattice spacing. In three dimensions the divergence is $a^{-2}$. However any $N$-body fixed-point distribution $\left|F_{\mathbf{R}}\right|^{2}$ must vanish as $\left|\vec{R}_{i}-\vec{R}_{j}\right|^{2}$ due to antisymmetry with respect to exchange of $\vec{r}_{i}$ and $\vec{r}_{j}$ in

$$
\left\langle\mathbf{r}\left|\bigwedge_{j=1, \cdots, N}\right| f_{j}(\mathbf{R})\right\rangle .
$$

This is enough to remove the clustering instability for $d \leq 3$. Therefore the continuum limit is well defined for the lowest filling approximation to the ground state energy,

$$
E_{N, N}^{0} \approx \sum_{\mathbf{R}}\left|F_{\mathbf{R}}\right|^{2} \sum_{j=1, \cdots, N} E_{j}(\mathbf{R})
$$

where $\left|F_{\mathbf{R}}\right|^{2}$ is an $N$-body fixed-point density. 


\section{Lowest filling approximation for the four-body system in three dimensions}

We test how well the lowest filling approximation works for the unpolarized four-body system in three dimensions. Again the coupling is tuned to the unitarity point and the lattice volume is $4^{3}$. Once we project onto the total momentum $\vec{P}=0$ subspace, there are 35 independent configurations $\mathbf{R}$ for the up spins. Similarly there are 35 independent configurations for the down spin positions $\mathbf{r}$. We construct a $35 \times 35$ matrix $M(\mathbf{r}, \mathbf{R})$ with elements

$$
M(\mathbf{r}, \mathbf{R})=\left|\left\langle\mathbf{r}\left|\bigwedge_{j=1,2}\right| f_{j}(\mathbf{R})\right\rangle\right|^{2},
$$

where $\left|f_{1}(\mathbf{R})\right\rangle,\left|f_{2}(\mathbf{R})\right\rangle$ are the lowest orbitals for $\mathbf{R}$. We then solve for the $N$-body fixedpoint density $\left|F_{\mathbf{R}}\right|^{2}$ satisfying

$$
\sum_{\mathbf{R}} M(\mathbf{r}, \mathbf{R})\left|F_{\mathbf{R}}\right|^{2}=\left|F_{\mathbf{r}}\right|^{2} .
$$

With $\left|F_{\mathbf{R}}\right|^{2}$ the ground state energy can be calculated using

$$
E_{2,2}^{0} \approx \sum_{\mathbf{R}}\left|F_{\mathbf{R}}\right|^{2} \sum_{j=1,2} E_{j}(\mathbf{R}) .
$$

We can also compute two-body correlation functions. The same-spin correlation function in the lowest filling approximation is

$$
\begin{aligned}
G_{\text {same }}(\vec{r}) & =\sum_{\vec{r}^{\prime}}\left\langle\Psi_{2,2}^{0}\left|: \rho_{\downarrow}\left(\vec{r}+\vec{r}^{\prime}\right) \rho_{\downarrow}\left(\vec{r}^{\prime}\right):\right| \Psi_{2,2}^{0}\right\rangle \\
& =\sum_{\vec{r}^{\prime}}\left\langle\Psi_{2,2}^{0}\left|: \rho_{\uparrow}\left(\vec{r}+\vec{r}^{\prime}\right) \rho_{\uparrow}\left(\vec{r}^{\prime}\right):\right| \Psi_{2,2}^{0}\right\rangle \\
& \approx \sum_{\mathbf{R}}\left(\delta_{\vec{R}_{1}, \vec{r}+\vec{R}_{2}}+\delta_{\vec{R}_{2}, \vec{r}+\vec{R}_{1}}\right)\left|F_{\mathbf{R}}\right|^{2} .
\end{aligned}
$$

In our dimensionless lattice normalization the same-spin correlation function summed over $\vec{r}$ equals 2. Also the opposite-spin correlation function is given by

$$
\begin{aligned}
G_{\mathrm{opp}}(\vec{r}) & =\sum_{\vec{r}^{\prime}}\left\langle\Psi_{2,2}^{0}\left|: \rho_{\uparrow}\left(\vec{r}+\vec{r}^{\prime}\right) \rho_{\downarrow}\left(\vec{r}^{\prime}\right):\right| \Psi_{2,2}^{0}\right\rangle \\
& =\sum_{\vec{r}^{\prime}}\left\langle\Psi_{2,2}^{0}\left|: \rho_{\downarrow}\left(\vec{r}+\vec{r}^{\prime}\right) \rho_{\uparrow}\left(\vec{r}^{\prime}\right):\right| \Psi_{2,2}^{0}\right\rangle \\
& \approx \sum_{\mathbf{R}} \sum_{j=1,2}\left[\left|f_{j}\left(\vec{r}+\vec{R}_{1}, \mathbf{R}\right)\right|^{2}+\left|f_{j}\left(\vec{r}+\vec{R}_{2}, \mathbf{R}\right)\right|^{2}\right]\left|F_{\mathbf{R}}\right|^{2} .
\end{aligned}
$$



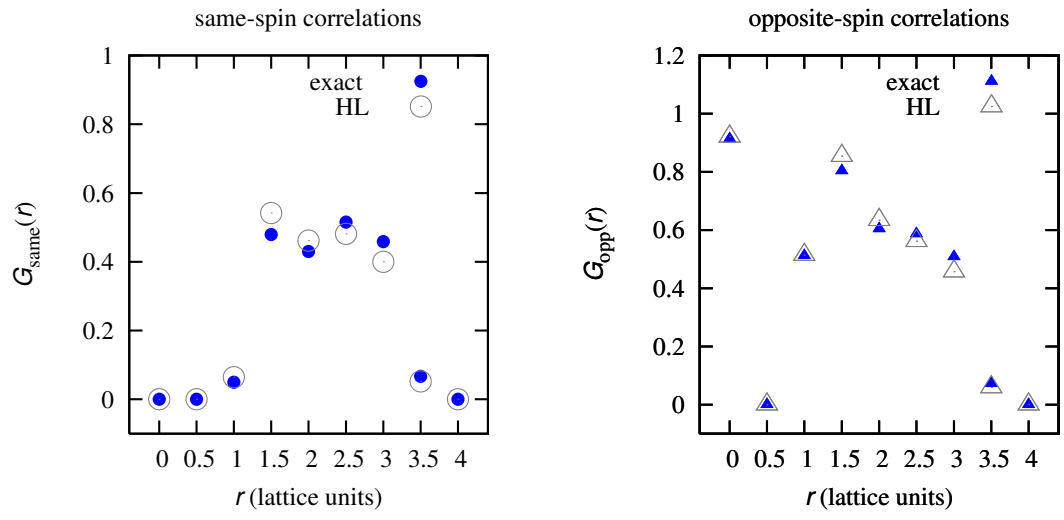

FIG. 3: Comparison of exact and heavy-light results for the same-spin and opposite-spin correlation functions. The data is binned by radial distance in lattice units rounded to the nearest half integer.

The opposite-spin correlation function summed over $\vec{r}$ equals 4 . Fig. 3 shows a comparison of exact results and heavy-light results in the lowest filling approximation for the same-spin and opposite-spin correlation functions. The exact results are Lanczos calculations for the ground state of $H$. The data is binned by radial distance $r=|\vec{r}|$ in lattice units rounded to the nearest half integer. The agreement between exact and heavy-light results for the correlation functions is rather good. The agreement for the ground state energy is also quite good. The exact value for the ratio $E_{2,2}^{0} / E_{2,2}^{0, \text { free }}$ is 0.138 while the heavy-light result gives 0.128. This level of quantitative agreement may seem surprising given the simplicity of the lowest filling approximation to the heavy-light ansatz. The method simply patches together a collection of single-particle orbitals using the $N$-body fixed-point constraint in Eq. (64). However the method appears to accurately describe the competition between the short-range attractive interaction and Fermi repulsion. We now check whether the agreement is also good for different interaction strengths.

The ratio $E_{N, N}^{0} / E_{N, N}^{0, \text { free }}$ tends towards negative infinity at strong attractive coupling and is therefore somewhat unwieldy to plot over a large range of coupling strengths. Let us define a different dimensionless ratio which is convenient for the attractive Hubbard model at arbitrary coupling and arbitrary dimensions. At fixed lattice volume $L^{d}$ we define the 
ratio

$$
e_{N, N}=\frac{E_{N, N}^{0}-E_{N, N}^{0, \text { free }}}{N\left|E_{1,1}^{0}\right|+E_{N, N}^{0, \text { free }}},
$$

where $E_{1,1}^{0}$ is the ground state energy for the two-body dimer in the same lattice volume $L^{d}$. In the limit of weak attractive coupling, $e_{N, N}$ tends towards 0 from below. In this case the physics is dominated by Fermi repulsion, and the ground state is similar to that of a free Fermi gas. In the limit of strong attractive coupling, $e_{N, N}$ tends towards -1 from above. Here the ground state consists of tightly-bound dimers with only weak interactions between dimers. For $e_{N, N}$ in the midrange between 0 and -1 , the competition between attractive interaction and Fermi repulsion is stalemated to some intermediate balance point. The unitarity limit in three dimensions fits this category. In the limit of large $N$ at unitarity the $N\left|E_{1,1}^{0}\right|$ term can be neglected relative to $E_{N, N}^{0, \text { free }}$. In this case

$$
e_{N, N} \rightarrow \frac{E_{N, N}^{0}-E_{N, N}^{0, \text { free }}}{E_{N, N}^{0, \text { free }}}=E_{N, N}^{0} / E_{N, N}^{0, \text { free }}-1 .
$$

In Fig. 4 we show results for $e_{2,2}$ for lattice lengths $L=3,4,5$ and various couplings. We express the coupling as a ratio of Hubbard model parameters

$$
U / t=2 m C .
$$

The plot on the left shows exact results computed using the Lanczos method. The unitarity point corresponds with $U / t=-7.914$, and the signal of scale invariance at unitarity can be seen by the agreement in $e_{2,2}$ for different $L$. The plot on the right shows the difference between heavy-light results in the lowest filling approximation and exact Lanczos results. From weak coupling at $e_{2,2} \approx 0$ to strong coupling at $e_{2,2} \approx-1$, the error of the heavy-light calculation is bounded by 0.02 . The method appears to accurately describe the crossover from four weakly-interacting fermions to a condensed pair of bosonic dimers.

\section{MARKOV CHAIN FOR $N$-BODY FIXED-POINT DENSITIES}

In this section we design a Markov chain process which generates the $N$-body fixed-point density $\left|F_{\mathbf{R}}\right|^{2}$ that solves the constraint in Eq. (64). Our construction establishes both existence and uniqueness. Let us define

$$
\Upsilon(\mathbf{r}, \mathbf{R})=\left|\left\langle\mathbf{r}\left|\bigwedge_{j=1, \cdots, N}\right| f_{j}(\mathbf{R})\right\rangle\right|^{2} .
$$



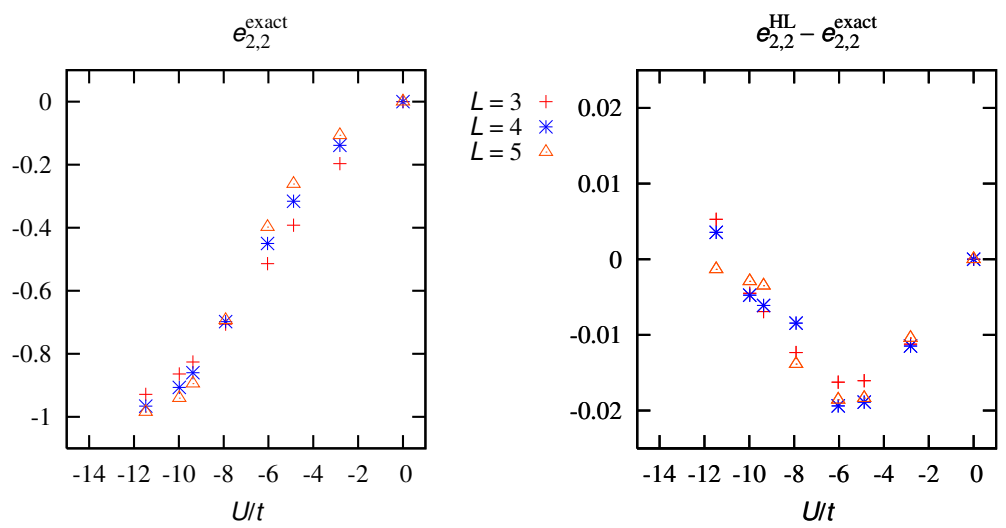

FIG. 4: Comparison of four-body exact Lanczos results, $e_{2,2}^{\text {exact}}$, and heavy-light results, $e_{2,2}^{\mathrm{HL}}$, for $L=3,4,5$ lattices in three dimensions plotted versus coupling $U / t=2 m C$.

We note that $\Upsilon(\mathbf{r}, \mathbf{R}) \geq 0$ for every pair $\mathbf{r}, \mathbf{R}$. Also for each $\mathbf{R}$,

$$
\sum_{\mathbf{r}} \Upsilon(\mathbf{r}, \mathbf{R})=1
$$

We define a Markov chain

$$
\mathbf{R}^{(0)} \rightarrow \mathbf{R}^{(1)} \rightarrow \cdots \rightarrow \mathbf{R}^{(k)} \rightarrow \mathbf{R}^{(k+1)} \rightarrow \cdots
$$

with transition probability

$$
p\left(\mathbf{R}^{(k+1)} \mid \mathbf{R}^{(k)}\right)=\Upsilon\left(\mathbf{R}^{(k+1)}, \mathbf{R}^{(k)}\right) .
$$

Each state in the Markov chain is a set of $N$ distinct $d$-dimensional vectors on an $L^{d}$ lattice. We note that

$$
\Upsilon\left(\mathbf{R}^{(k+1)}, \mathbf{R}^{(k)}\right)=0
$$

if and only if $\left|\mathbf{R}^{(k+1)}\right\rangle$ and $\bigwedge_{j=1, \cdots, N}\left|f_{j}\left(\mathbf{R}^{(k)}\right)\right\rangle$ are exactly orthogonal. Since the state space is finite the possibility of accidental orthogonality between $\left|\mathbf{R}^{(k+1)}\right\rangle$ and $\bigwedge_{j=1, \cdots, N}\left|f_{j}\left(\mathbf{R}^{(k)}\right)\right\rangle$ is a set of measure zero and can avoided by an arbitrarily small change in the coupling $C$. However it is also possible that the orthogonality arises from some mismatch of exactly conserved quantum numbers. These quantum numbers would be associated with some symmetry subgroup of the lattice shared by $\mathbf{R}^{(k+1)}$ and $\mathbf{R}^{(k)}$. It might be a reflection symmetry, 
rotational symmetry, translational symmetry, or some combination of each. However for large $L$ the relative proportion of such symmetric configurations $\mathbf{R}^{(k+1)}$ and $\mathbf{R}^{(k)}$ is suppressed by powers of $L$ and therefore exceedingly rare. We let $\epsilon$ be a small positive number and define a modified chain with transition probability matrix

$$
\begin{gathered}
p\left(\mathbf{R}^{(k+1)} \mid \mathbf{R}^{(k)}\right)=\Upsilon_{\epsilon}\left(\mathbf{R}^{(k+1)}, \mathbf{R}^{(k)}\right), \\
\Upsilon_{\epsilon}(\mathbf{r}, \mathbf{R})=\frac{\max [\Upsilon(\mathbf{r}, \mathbf{R}), \epsilon]}{\sum_{\mathbf{r}} \max [\Upsilon(\mathbf{r}, \mathbf{R}), \epsilon]}
\end{gathered}
$$

For any $\mathbf{R}^{\prime}$ and $\mathbf{R}^{\prime \prime}$ there exists some chain of finite length from $\mathbf{R}^{\prime}$ to $\mathbf{R}^{\prime \prime}$ such that the transition probability at each step is nonzero. In fact we can get there in only one step. Hence the Markov chain is ergodic and there exists a unique invariant distribution, call it $\left|F_{\mathbf{R}}\right|^{2}$, such that

$$
\sum_{\mathbf{R}} \Upsilon_{\epsilon}\left(\mathbf{R}^{\prime}, \mathbf{R}\right) \times\left|F_{\mathbf{R}}\right|^{2}=\left|F_{\mathbf{R}^{\prime}}\right|^{2}
$$

We take the limit as $\epsilon \rightarrow 0$ and obtain $\left|F_{\mathbf{R}}\right|^{2}$ as the unique $N$-body fixed-point density.

This fixed-point Markov chain shares some features with iterative solutions of the KohnSham equations in density functional theory [29]. Both involve finding the lowest orbitals of a Schrödinger potential which in turn depends on the particle densities. However there are important differences between this method and the Kohn-Sham equations. The fixed-point Markov chain presented above solves for $N$-body densities rather than one-body densities. Also in contrast with density functional theory, this method yields an ab initio solution for the uniform system. We discuss possible extensions to non-uniform systems in the discussion section.

As a precise test of the Markov chain algorithm for $\left|F_{\mathbf{R}}\right|^{2}$ we compare with heavy-light calculations presented earlier for the unpolarized four-body system at unitarity on a $4^{3}$ lattice. For this calculation and all other heavy-light calculations presented in the following we generate the fixed-point $\left|F_{\mathbf{R}}\right|^{2}$ using 4000 steps of the Markov chain

$$
\mathbf{R}^{(0)} \rightarrow \mathbf{R}^{(1)} \rightarrow \cdots \rightarrow \mathbf{R}^{(k)} \rightarrow \mathbf{R}^{(k+1)} \rightarrow \cdots
$$

with transition probability

$$
p\left(\mathbf{R}^{(k+1)} \mid \mathbf{R}^{(k)}\right)=\Upsilon\left(\mathbf{R}^{(k+1)}, \mathbf{R}^{(k)}\right) .
$$



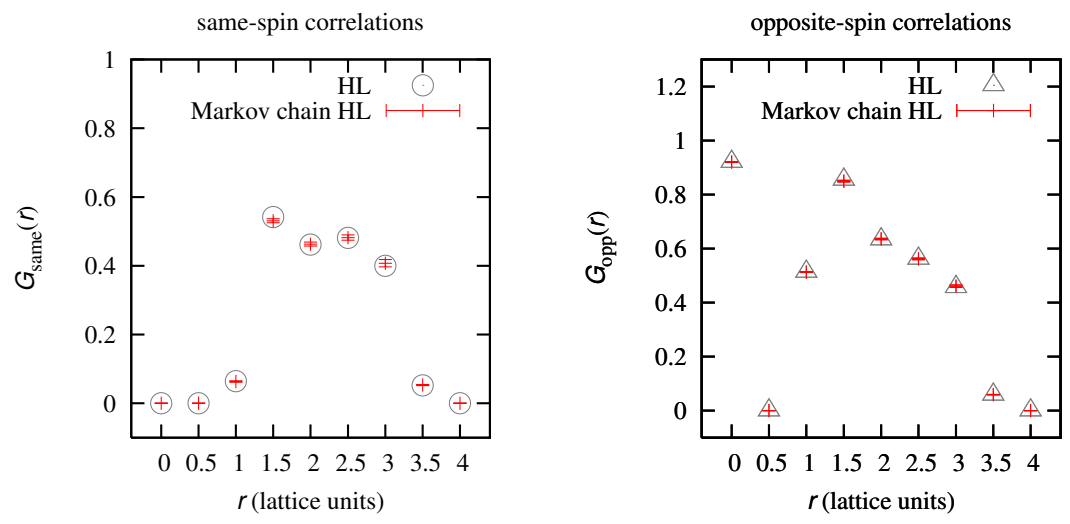

FIG. 5: Comparison of Markov chain and direct heavy-light calculations for the same-spin and opposite-spin correlation functions

Each step of the chain $\mathbf{R}^{(k)} \rightarrow \mathbf{R}^{(k+1)}$ is produced using a Metropolis algorithm with 100 updates per up-spin particle location according to the probability distribution

$$
\Upsilon\left(\mathbf{R}, \mathbf{R}^{(k)}\right)=\left|\left\langle\mathbf{R}\left|\bigwedge_{j=1, \cdots, N}\right| f_{j}\left(\mathbf{R}^{(k)}\right)\right\rangle\right|^{2} .
$$

The entire calculation is repeated several times with different random number seeds and the results are averaged. The standard deviation of the distribution is used to determine the stochastic error of the average.

In Fig. 5 we show a comparison of calculations for the same-spin and opposite-spin correlations functions using the Markov chain algorithm and the direct calculations shown previously in Fig. 3. The error bars shown are estimated stochastic errors. We see that the Markov chain algorithm reproduces the direct calculations for the correlation functions with no detectable systematic error. The Markov chain heavy-light result for $E_{2,2}^{0} / E_{2,2}^{0 \text {,free }}$ is $0.127(1)$, which agrees with the direct heavy-light calculation of 0.128 . The advantage of the Markov chain algorithm is that the computational scaling for large systems is very favorable, nearly linear in the lattice volume and number of particles. 


\section{FEW-BODY SYSTEMS IN ONE AND TWO DIMENSIONS}

In this section we compare heavy-light calculations for $e_{N, N}$ in the lowest filling approximation with Lanczos calculations for few-body systems in one and two spatial dimensions. The rapid computational scaling of the Lanczos calculation, $L^{(2 N-1) d}$, limits the system sizes for which the comparison is possible. Let us first discuss the properties of bound dimers in the $1 \mathrm{D}, 2 \mathrm{D}$, and 3D attractive Hubbard models.

In one dimension at infinite volume the dimer has binding energy [27]

$$
B_{2}=-E_{1,1}^{0}=\frac{m C^{2}}{4}+\cdots,
$$

and characteristic size

$$
\frac{1}{\sqrt{m B_{2}}}=\frac{2}{m|C|}+\cdots
$$

The ellipses denote lattice spacing corrections which become important when the characteristic size is not much larger than one lattice spacing. In two dimensions at infinite volume the dimer has binding energy [30]

$$
B_{2}=-E_{1,1}^{0}=\frac{3.24 \pi^{2}}{m} \exp \left(\frac{4 \pi}{m C}\right)+\cdots
$$

and characteristic size

$$
\frac{1}{\sqrt{m B_{2}}}=\frac{1}{1.80 \pi} \exp \left(-\frac{2 \pi}{m C}\right)+\cdots
$$

In three dimensions at infinite volume and $m C<-3.957$, the dimer has binding energy [19, 21, 23]

$$
B_{2}=-E_{1,1}^{0}=\frac{1}{m a_{\mathrm{scatt}}^{2}}=\frac{16 \pi^{2}}{m}\left(\frac{1}{m C}+\frac{1}{3.957}\right)^{2}+\cdots,
$$

and characteristic size

$$
\frac{1}{\sqrt{m B_{2}}}=\frac{1}{4 \pi\left(\frac{1}{m C}+\frac{1}{3.957}\right)}+\cdots
$$

In one and two dimensions the attractive Hubbard model has no nontrivial scale-invariant point analogous to the unitarity point in three dimensions.

Fig. 6] shows $e_{2,2}$ for the unpolarized four-body system in one dimension. Fig. 7 shows

$e_{3,3}$ for the unpolarized six-body system in one dimension. For both the four-body and six-body systems the error of the heavy-light result is smaller than 0.015. We note that the six-body heavy-light data for $L=4$ is exact. This is because the system can be regarded as the ground state of one up-spin hole and one down-spin hole. Similar to the unpolarized 

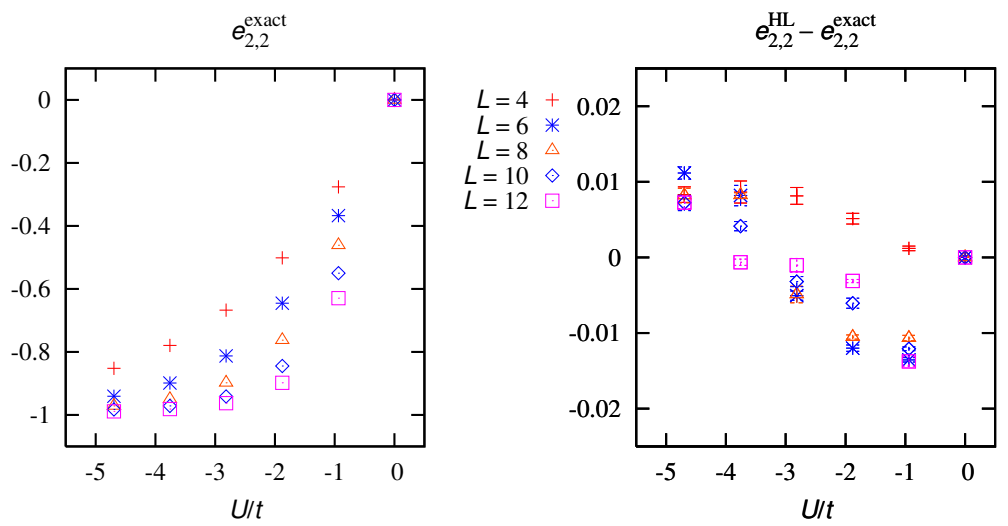

FIG. 6: Comparison of four-body exact Lanczos results, $e_{2,2}^{\text {exact }}$, and heavy-light results, $e_{2,2}^{\mathrm{HL}}$, for $L=4,6,8,10,12$ lattices in one dimension plotted versus coupling $U / t=2 m C$.
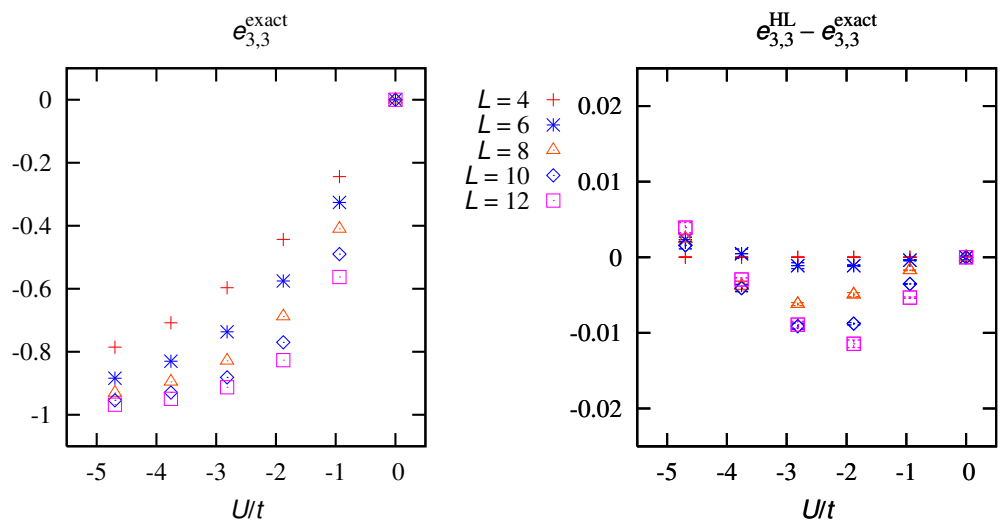

FIG. 7: Comparison of six-body exact Lanczos results, $e_{3,3}^{\text {exact }}$, and heavy-light results, $e_{3,3}^{\mathrm{HL}}$, for $L=4,6,8,10,12$ lattices in one dimension plotted versus coupling $U / t=2 m C$.

two-particle system, the ground state of the unpolarized two-hole system depends only on the reduced mass $\mu$,

$$
\frac{1}{\mu}=\frac{1}{m_{\uparrow}}+\frac{1}{m_{\downarrow}}
$$

which is unaltered in the heavy-light formalism.

Just as in the three-dimensional example at unitarity, the method appears to accurately 

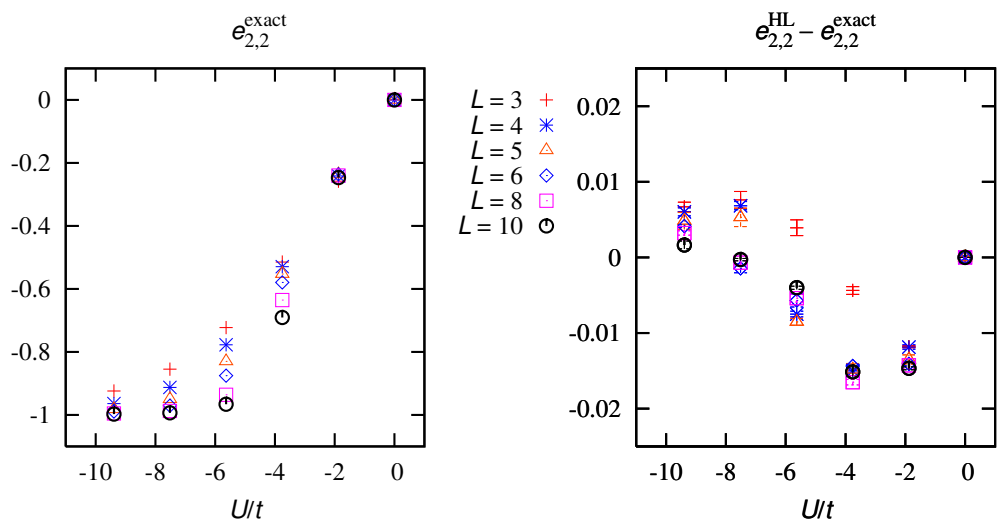

FIG. 8: Comparison of four-body exact Lanczos results, $e_{2,2}^{\text {exact }}$, and heavy-light results, $e_{2,2}^{\mathrm{HL}}$, for $L=3,4,5,6,8,10$ lattices in two dimensions plotted versus coupling $U / t=2 m C$.

describe the crossover from fermions to condensed bosonic dimers in one dimension. The difference between the ground state energy $E_{N, N}^{0}$ and $N$ times the dimer energy $E_{1,1}^{0}$ can be viewed as Fermi repulsion of overlapping of dimer wavefunctions. In one dimension the dimer wavefunction has an exponential tail proportional to

$$
\exp \left(\frac{1}{2} m C|x|\right)=\exp \left(\frac{U}{4 t}|x|\right) \text {. }
$$

This simple picture is consistent with the exponential dependence of $e_{N, N}$ on $U / t$ seen in both the four-body and six-body results for large negative $U / t$.

Fig. 8 shows $e_{2,2}$ for the unpolarized four-body system in two dimensions. Fig. 9 shows $e_{3,3}$ for the unpolarized six-body system in two dimensions. In both cases the error of the heavy-light result is bounded by 0.02. Just as in the one- and three-dimensional examples, the method appears to accurately describe the crossover from fermions to condensed bosonic dimers. In two dimensions the dimer wavefunction at infinite volume has an exponential tail with characteristic length

$$
\frac{1}{\sqrt{m B_{2}}}=\frac{1}{1.80 \pi} \exp \left(-\frac{2 \pi}{m C}\right)=\frac{1}{1.80 \pi} \exp \left(-\frac{4 \pi}{U / t}\right) \text {. }
$$

The dimer increases its size dramatically for small negative $U / t$. But at finite volume it eventually wraps around the periodic boundary at distance $L$. This boundary effect changes 

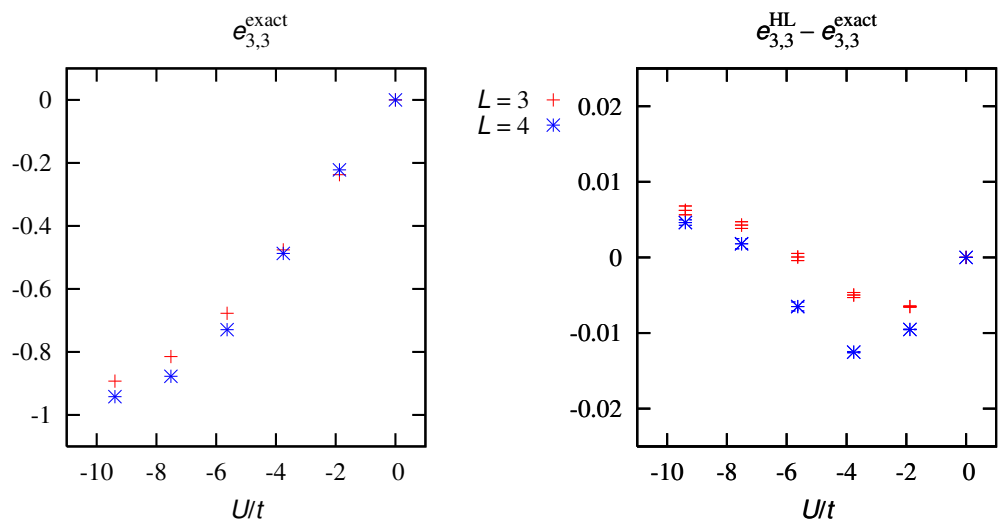

FIG. 9: Comparison of six-body exact Lanczos results, $e_{3,3}^{\text {exact }}$, and heavy-light results, $e_{3,3}^{\mathrm{HL}}$, for $L=3,4$ lattices in two dimensions plotted versus coupling $U / t=2 m C$.

the behavior of $e_{N, N}$. This can be seen as a crossover to approximately linear dependence on $U / t$ for $U / t \gtrsim-4$ in both the four-body and six-body results.

\section{DIMER-DIMER SCATTERING IN THREE DIMENSIONS}

We have already presented results for $e_{2,2}$ in three dimensions. In this section we reanalyze the data to extract low-energy scattering parameters for dimer-dimer scattering. Lüscher's formula [31, 32, 33, 34] relates the energy levels for any two-body system in a finite periodic cube to the S-wave phase shift. In the appendix we discuss how to use Lüscher's formula in the two-body system with one up spin and one down spin to measure the fermion-fermion scattering length, $a_{\text {scatt }}$. At strong coupling we can also use Lüscher's formula in the four-body system to measure the S-wave dimer-dimer phase shift. When $L$ is much bigger than $a_{\text {scatt }}$ we can interpret the energy difference

$$
\Delta E_{2,2}^{0}=E_{2,2}^{0}-2 E_{1,1}^{0}
$$

as the energy of the two-dimer system relative to threshold. We then determine the dimerdimer phase shift using

$$
p_{\mathrm{D}} \cot \delta_{\mathrm{DD}}=\frac{1}{\pi L} S(\eta), \quad \eta=\left(\frac{L p_{\mathrm{D}}}{2 \pi}\right)^{2}
$$




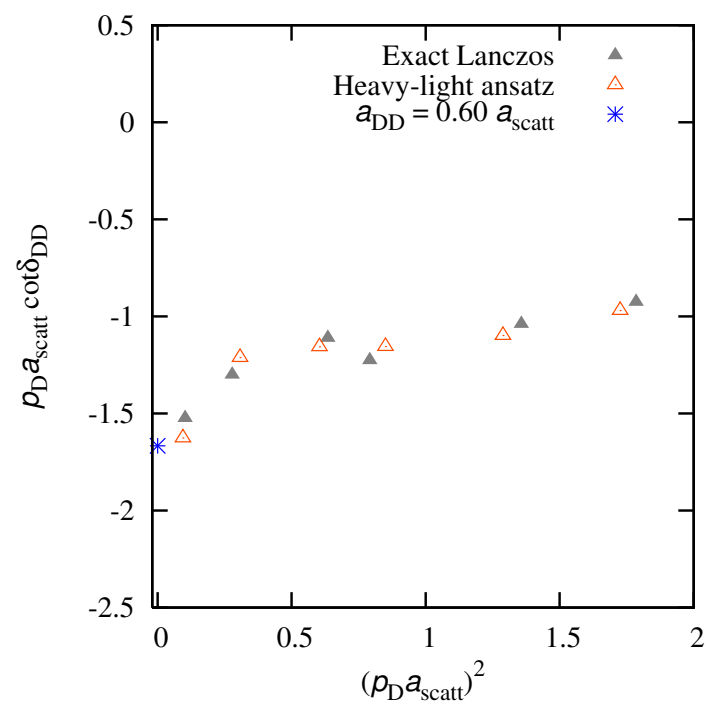

FIG. 10: Results for $p_{\mathrm{D}} \cot \delta_{\mathrm{DD}}$ as a function of the dimer momentum squared. Both quantities are measured in units of $a_{\text {scatt }}$. For comparison we also show the limit of $p_{\mathrm{D}} \cot \delta_{\mathrm{DD}}$ at zero momentum determined by the result $a_{\mathrm{DD}} \approx 0.60 a_{\text {scatt }}$.

where $p_{\mathrm{D}}$ is the dimer momentum, $S(\eta)$ is the three-dimensional zeta function defined in the appendix, and $\delta_{\mathrm{DD}}$ is the dimer-dimer S-wave phase shift. It is convenient to measure everything in units of the fermion-fermion scattering length $a_{\text {scatt }}$.

Results for $p_{\mathrm{D}} \cot \delta_{\mathrm{DD}}$ are shown in Fig. 10 as a function of dimer momentum squared. For comparison we also indicate the result $a_{\mathrm{DD}} \approx 0.60 a_{\text {scatt }}[35,36]$ which determines $p_{\mathrm{D}} \cot \delta_{\mathrm{DD}}$ at zero momentum using the effective range expansion,

$$
p_{\mathrm{D}} \cot \delta_{\mathrm{DD}} \approx-\frac{1}{a_{\mathrm{DD}}}+\frac{1}{2} r_{\mathrm{DD}} p_{\mathrm{D}}^{2}+\cdots .
$$

We see in Fig. 10 that the heavy-light and exact Lanczos results agree at the level of a few percent level. Also both agree with the dimer-dimer scattering length result $a_{\mathrm{DD}} \approx$ $0.60 a_{\text {scatt }}$. From this plot we can also estimate the dimer-dimer effective range,

$$
r_{\mathrm{DD}} \approx 2.6 a_{\text {scatt }} .
$$




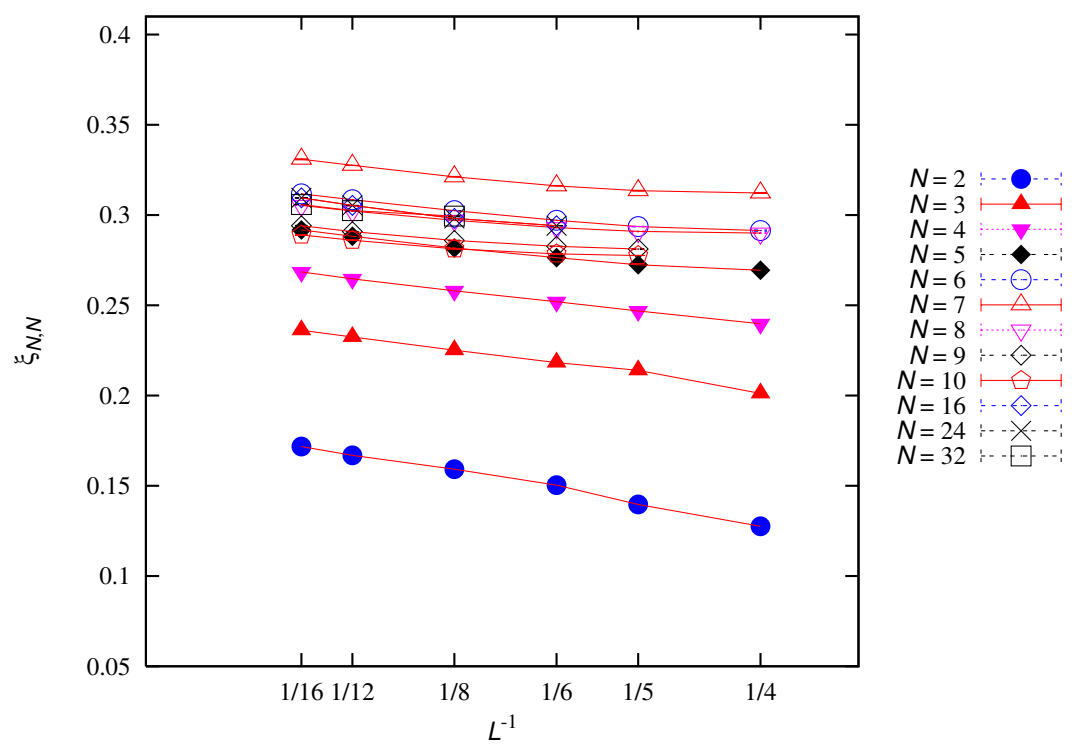

FIG. 11: Heavy-light results for $\xi_{N, N}$ for $N$ from 2 to 32 . The results are plotted versus $L^{-1}$, where $L$ ranges from 4 to 16 .

\section{BCS-BEC CROSSOVER IN THREE DIMENSIONS}

\section{A. Many-body results at unitarity}

We present symmetric heavy-light results in the lowest filling approximation at unitarity for

$$
\xi_{N, N}=\frac{E_{N, N}^{0}}{E_{N, N}^{0, \text { free }}}
$$

for a wide range of values for $N$ and $L$. Fig. 11 shows results for $\xi_{N, N}$ for the four-body system, $N=2$, up to the sixty-four-body system, $N=32$. The results are plotted versus $L^{-1}$, where $L$ ranges from 4 to 16 . The dependence on $L$ is relatively mild. The maximum for $\xi_{N, N}$ for $N=7$ appears to be caused by the closed shell at $N=7$ for the free Fermi system in a periodic cube. In the continuum and thermodynamic limits $L, N \rightarrow \infty$ we find

$$
\xi=\lim _{L, N \rightarrow \infty} \xi_{N, N}=0.31(1)
$$

The heavy-light results can be compared with published Euclidean lattice Monte Carlo 
results at small volumes shown in Table 1 [22].

Table 1: $\xi_{N, N}$ using Euclidean lattice Monte Carlo

\begin{tabular}{|l|l|l|l|l|}
\hline$N=3$ & $N=5$ & $N=7$ & $N=9$ & $N=11$ \\
\hline $0.19(2)$ & $0.24(2)$ & $0.28(2)$ & $0.23(2)$ & $0.25(2)$ \\
\hline
\end{tabular}

These results correspond with an average of data from $L=4,5,6$ for each $N=3,5,7,9$ and $L=5,6$ for $N=11$. For the same $N$ and $L$, the heavy-light results in Fig. 11] agree reasonably well with the Euclidean Monte Carlo data, with a relative difference of at most $20 \%$.

The heavy-light data for larger values of $L$ probe much further towards the continuum limit. These results suggest that lattice cutoff effects cannot explain the discrepancy between lattice Monte Carlo results and continuum fixed-node Monte Carlo results for the same values of $N$. Fixed-node Green's function Monte Carlo calculations have found $\xi_{N, N}$ to be $0.44(1)$ [37, 38] and 0.42(1) [39] for comparable values of $N$. Further studies are needed to determine if different nodal surfaces can produce lower ground state energies in fixed-node calculations. The symmetric heavy-light ansatz may be useful in probing this question more deeply. In addition to the aforementioned lattice Monte Carlo and fixed-node simulations there are a number of other theoretical calculations [19, 40, 41, 42, 43, 44, 45, 46, 47, 48, 49, 50, 51, 52, 53] and experimental measurements of $\xi[4,7,8,9,10,54]$ which span the range from about 0.2 to 0.6 .

\section{B. Results for general scattering length}

We consider $\xi_{N, N}$ as a function of $k_{F} a_{\text {scatt }}$. In the limit of strong attractive coupling and the thermodynamic limit $N \rightarrow \infty$ at fixed density, we get

$$
\xi=\lim _{N \rightarrow \infty} \frac{E_{N, N}^{0}}{E_{N, N}^{0, \text { free }}}=\lim _{N \rightarrow \infty} \frac{N E_{1,1}^{0}+\frac{N(N-1)}{2} \frac{4 \pi a_{\mathrm{DD}}}{2 m L^{3}}}{2 \times \frac{3}{5} N E_{F}}+O\left(k_{F}^{2} a_{\text {scatt }}^{2}\right),
$$

where $E_{1,1}^{0}$ is the energy for one dimer and $a_{\mathrm{DD}}$ is the dimer-dimer scattering length. The first term takes into account the binding energy of the dimer while the second gives the contribution due to dimer-dimer interactions. Although we have written the expansion in powers of $k_{F} a_{\text {scatt }}$, a more accurate estimate of the appropriate expansion parameter is $a_{\text {scatt }}$ divided by the average spacing between particles $d \approx\left(6 \pi^{2}\right)^{1 / 3} k_{F}^{-1}$. We expect the $O\left(k_{F}^{2} a_{\text {scatt }}^{2}\right)$ error to be small for $k_{F} a_{\text {scatt }} \lesssim 2$. 
For a periodic cube we have

$$
E_{F}=\frac{1}{2 m}\left(6 \pi^{2} \frac{N}{L^{3}}\right)^{2 / 3}=\frac{\left(6 \pi^{2} N\right)^{2 / 3}}{2 m L^{2}}
$$

and

$$
k_{F}=\frac{\left(6 \pi^{2} N\right)^{1 / 3}}{L} .
$$

In the continuum limit the energy for one dimer is

$$
E_{1,1}^{0}=-\frac{1}{m a_{\text {scatt }}^{2}}
$$

and the dimer-dimer scattering length is approximately $a_{\mathrm{DD}} \approx 0.60 a_{\text {scatt }}$. Putting these together we get

$$
\xi=\lim _{N \rightarrow \infty} \frac{E_{N, N}^{0}}{E_{N, N}^{0, \text { free }}}=-\frac{5}{3 k_{F}^{2} a_{\text {scatt }}^{2}}+0.60 \times \frac{5}{18 \pi} k_{F} a_{\text {scatt }}+O\left(k_{F}^{2} a_{\text {scatt }}^{2}\right) .
$$

We refer to the first two terms in this expansion as leading order (LO) and next-to-leading order (NLO) in the strong-coupling expansion.

In the weak-coupling limit we have [55, 56]

$$
\xi_{N, N}=\frac{E_{N, N}^{0}}{E_{N, N}^{0, \text { free }}}=1+\frac{10}{9 \pi} k_{F} a_{\text {scatt }}+\frac{4(11-2 \ln 2)}{21 \pi^{2}} k_{F}^{2} a_{\text {scatt }}^{2}+O\left(k_{F}^{3} a_{\text {scatt }}^{3}\right) .
$$

We refer to the first three terms in this expansion as leading order (LO), next-to-leading order (NLO), and next-to-next-to-leading order (NNLO) in the weak-coupling expansion. In Fig. 12 we show $\xi_{N, N}$ as a function of $k_{F}^{-1} a_{\text {scatt }}^{-1}$ for $N=32$ and $L=16$ using the symmetric heavy-light ansatz in the lowest filling approximation. For comparison we show the analytic strong-coupling and weak-coupling results.

We see that the heavy-light results are very close to the strong-coupling results for $k_{F}^{-1} a_{\text {scatt }}^{-1} \gtrsim 0.3$. The lowest filling approximation is also not bad in the weak-coupling limit for $k_{F}^{-1} a_{\text {scatt }}^{-1} \lesssim-1$. Near the unitarity point we can expand

$$
\xi_{N, N}=\frac{E_{N, N}^{0}}{E_{N, N}^{0, \text { free }}}=\xi-\xi_{1} k_{F}^{-1} a_{\text {scatt }}^{-1}-\xi_{2} k_{F}^{-2} a_{\text {scatt }}^{-2}+\cdots .
$$

For $N=32$ and $L=16$ we find by least squares fitting that

$$
\begin{gathered}
\xi=0.306(1), \\
\xi_{1}=0.805(2),
\end{gathered}
$$




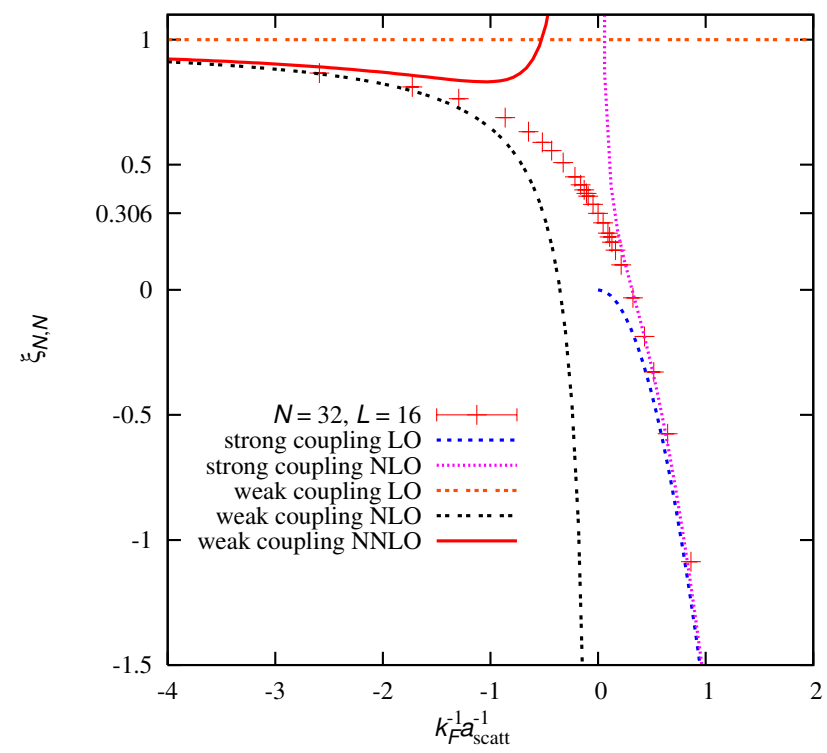

FIG. 12: Plot of $\xi_{N, N}$ for $N=32$ and $L=16$ as a function of $k_{F}^{-1} a_{\text {scatt }}^{-1}$. For comparison we show the analytic strong-coupling and weak-coupling results.

$$
\xi_{2}=0.63(3)
$$

The heavy-light result for $\xi_{1}$ is within $20 \%$ of a Euclidean lattice Monte Carlo calculation [25] which found $\xi_{1}=1.0(1)$. In contrast with $\xi$, there is general agreement in the recent literature on the value of $\xi_{1}$ calculated using different methods [38, 39, 52, 57].

\section{DISCUSSION}

\section{A. Lowest filling approximation and beyond}

In this analysis we have tested the symmetric heavy-light ansatz in the lowest filling approximation on numerous few- and many-body systems for the 1D, 2D, and 3D attractive Hubbard models. In each case the method appears to be accurate. It is perhaps not surprising that the lowest filling approximation is reliable at strong attractive coupling. In this limit the fermions form tightly-bound dimers which are weakly interacting and condense in the ground state. In the ground state the relative momentum between dimer pairs is relatively low, and the up-spin coordinates $\mathbf{R}$ can be viewed as surrogates for the locations of dimer pairs. The $N$-body fixed-point constraint sets $\left|F_{\mathbf{R}}\right|^{2}$ so that up spins and down spins 
are arranged in the same manner relative to neighboring particles. At very strong coupling $\left|F_{\mathbf{R}}\right|^{2}$ is essentially independent of $\mathbf{R}$, indicating that the dimers are non-interacting bosons each in the zero momentum state. As the attractive coupling becomes weaker, the size of the dimers increases and the effect of Fermi repulsion becomes more important.

For the systems we have considered here the lowest filling approximation works well at weak attractive coupling. However in future studies one may consider systematic improvements to the lowest filling approximation that include excited orbitals. This improvement would probably be needed to study the repulsive Hubbard model where there is no gap between the lowest $N$ orbitals and higher orbitals. In the lowest filling approximation the coefficients $\left|F_{\mathbf{R}}\right|^{2}$ are set by imposing $Z_{2}$ symmetry on the same-spin correlations,

$$
\left\langle\Phi_{N, N}\left|: \rho_{\uparrow}\left(\vec{r}_{1}\right) \times \cdots \times \rho_{\uparrow}\left(\vec{r}_{N}\right):\right| \Phi_{N, N}\right\rangle=\left\langle\Phi_{N, N}\left|: \rho_{\downarrow}\left(\vec{r}_{1}\right) \times \cdots \times \rho_{\downarrow}\left(\vec{r}_{N}\right):\right| \Phi_{N, N}\right\rangle .
$$

If excited orbitals are included, a larger set of coefficients $\left|F_{\mathbf{R},\left\{j_{n}\right\}}\right|^{2}$ must be determined. These can set by imposing $Z_{2}$ symmetry on mixed-spin correlations such as

$$
\begin{aligned}
\left\langle\Phi_{N, N}\right| & : \rho_{\uparrow}\left(\vec{r}_{1}\right) \times \rho_{\uparrow}\left(\vec{r}_{2}\right) \times \rho_{\downarrow}\left(\vec{r}_{3}\right):\left|\Phi_{N, N}\right\rangle \\
& =\left\langle\Phi_{N, N}\left|: \rho_{\downarrow}\left(\vec{r}_{1}\right) \times \rho_{\downarrow}\left(\vec{r}_{2}\right) \times \rho_{\uparrow}\left(\vec{r}_{3}\right):\right| \Phi_{N, N}\right\rangle .
\end{aligned}
$$

It is not clear that an algorithm can be designed to solve these constraints a priori, as we did for the lowest filling approximation. Therefore one approach would be to determine some parameterization for $\left|F_{\mathbf{R},\left\{j_{n}\right\}}\right|^{2}$ with unknown coefficients setting the relative weight of each orbital set $\left\{j_{n}\right\}$ for fixed $\mathbf{R}$. These coefficients could then be determined a posteriori by least squares fitting to the mixed-spin correlation constraints.

\section{B. R-commuting operators and phases}

We define an $\mathbf{R}$-commuting operator as any operator which commutes with the up-spin density $\rho_{\uparrow}(\vec{r})$ for all $\vec{r}$. Some examples of $\mathbf{R}$-commuting operators include $H_{\mathrm{HL}}$ and the up-spin and down-spin density operators. For any $\mathbf{R}$-commuting operator $O$ the ground state expectation value is

$$
\left\langle\Psi_{N, N}^{0}|O| \Psi_{N, N}^{0}\right\rangle=\sum_{\mathbf{R}} \sum_{\left\{j_{n}\right\},\left\{j_{n}^{\prime}\right\}} F_{\mathbf{R},\left\{j_{n}\right\}}^{*} F_{\mathbf{R},\left\{j_{n}^{\prime}\right\}} O_{\left\{j_{n}\right\},\left\{j_{n}^{\prime}\right\}}^{\mathbf{R}},
$$


where

$$
O_{\left\{j_{n}\right\},\left\{j_{n}^{\prime}\right\}}^{\mathbf{R}}=\left[\left\langle\mathbf{R}\left|\otimes \bigwedge_{n}\left\langle f_{j_{n}}(\mathbf{R})\right|\right]\left[|\mathbf{R}\rangle \otimes \bigwedge_{n}\left|f_{j_{n}^{\prime}}(\mathbf{R})\right\rangle\right] .\right.\right.
$$

Suppose $O$ is an R-commuting $M$-body operator with $M \ll N$. Due to the orthogonality of orbitals, the orbital sets $\left\{j_{n}\right\}$ and $\left\{j_{n}^{\prime}\right\}$ must be the same for all orbitals left untouched by $O$. Because of this constraint the diagonal elements $O_{\left\{j_{n}\right\},\left\{j_{n}\right\}}^{\mathbf{R}}$ are enhanced by powers of $N$ relative to the off-diagonal matrix elements $O_{\left\{j_{n}\right\},\left\{j_{n}^{\prime}\right\}}^{\mathbf{R}}$. For the special case $O=H_{\mathrm{HL}}$ all the off-diagonal matrix elements are in fact zero. If the weight of $\left|F_{\mathbf{R},\left\{j_{n}\right\}}\right|^{2}$ is dominated by orbital sets $\left\{j_{n}\right\}$ close to the lowest orbital filling $\{1, \cdots, N\}$, we can approximate $O_{\left\{j_{n}\right\},\left\{j_{n}\right\}}^{\mathbf{R}}$ by the diagonal element at lowest orbital filling,

$$
O_{\left\{j_{n}\right\},\left\{j_{n}\right\}}^{\mathbf{R}} \approx O_{\{1, \cdots, N\},\{1, \cdots, N\}}^{\mathbf{R}} .
$$

This leaves us with

$$
\left\langle\Psi_{N, N}^{0}|O| \Psi_{N, N}^{0}\right\rangle \approx \sum_{\mathbf{R}} O_{\{1, \cdots, N\},\{1, \cdots, N\}}^{\mathbf{R}} \sum_{\left\{j_{n}\right\}}\left|F_{\mathbf{R},\left\{j_{n}\right\}}\right|^{2} \approx \sum_{\mathbf{R}} O_{\{1, \cdots, N\},\{1, \cdots, N\}}^{\mathbf{R}}\left|F_{\mathbf{R}}\right|^{2} .
$$

The expectation values of $\mathbf{R}$-commuting operators are simple since we can keep only diagonal matrix elements, and the sign and phase of $F_{\mathbf{R}}$ is irrelevant. The expectation values of other operators are more challenging. One example that is not $\mathbf{R}$-commuting is the difermion pair correlation,

$$
\psi^{2 \dagger}(\vec{r}) \psi^{2}(\overrightarrow{0})
$$

where

$$
\psi^{2}(\vec{r})=a_{\uparrow}(\vec{r}) a_{\downarrow}(\vec{r}) .
$$

Here we need to compute matrix elements for orbitals from different up-spin configurations as well as nontrivial geometric phases which may arise going from one up-spin configuration $\mathbf{R}$ to another $\mathbf{R}^{\prime}$.

\section{Non-uniform systems}

The Markov chain algorithm for $N$-body fixed-point densities at lowest filling works also for the non-uniform case with external potential $V(\vec{r})$. Heavy-light calculations should be possible for systems such as harmonic traps used in cold atomic experiments. In the case of 
slowly-varying potentials these calculations could be compared with results obtained using density functional theory [58, 59, 60, 61, 62].

Let us define $H[V]$ with potential $V(\vec{r})$ as

$$
H[V]=H+\sum_{\vec{r}} V(\vec{r})\left[\rho_{\uparrow}(\vec{r})+\rho_{\downarrow}(\vec{r})\right]
$$

where $H$ is the attractive Hubbard Hamiltonian defined in Eq. (40). If $E_{N, N}^{0}[V]$ is the ground state energy of $H[V]$, the straightforward generalization of the symmetric heavy-light ansatz gives

$$
\min _{U\left|\Phi_{N, N}\right\rangle=\left|\Phi_{N, N}\right\rangle} \frac{\left\langle\Phi_{N, N}\left|H_{\mathrm{HL}}[V]\right| \Phi_{N, N}\right\rangle}{\left\langle\Phi_{N, N} \mid \Phi_{N, N}\right\rangle}=E_{N, N}^{0}[V]
$$

where

$$
H_{\mathrm{HL}}[V]=H_{\mathrm{HL}}+\sum_{\vec{r}} V(\vec{r})\left[\rho_{\uparrow}(\vec{r})+\rho_{\downarrow}(\vec{r})\right] .
$$

However we could also define a more general form for $H_{\mathrm{HL}}$,

$$
H_{\mathrm{HL}}\left[V, V_{A}\right]=H_{\mathrm{HL}}+\sum_{\vec{r}}\left[V(\vec{r})-V_{A}(\vec{r})\right] \rho_{\uparrow}(\vec{r})+\sum_{\vec{r}}\left[V(\vec{r})+V_{A}(\vec{r})\right] \rho_{\downarrow}(\vec{r}),
$$

for arbitrary $V_{A}(\vec{r})$. The contribution of $V_{A}(\vec{r})$ cancels from the expectation value in Eq. (120). For unpolarized systems adding an overall constant to the auxiliary potential $V_{A}(\vec{r})$ has no effect, and so we can set

$$
\sum_{\vec{r}} V_{A}(\vec{r})=0
$$

The optimal $V_{A}(\vec{r})$ can be found by minimizing the Rayleigh-Ritz ratio,

$$
\min _{U\left|\Phi_{N, N}\right\rangle=\left|\Phi_{N, N}\right\rangle} \frac{\left\langle\Phi_{N, N}\left|H_{\mathrm{HL}}\left[V, V_{A}\right]\right| \Phi_{N, N}\right\rangle}{\left\langle\Phi_{N, N} \mid \Phi_{N, N}\right\rangle},
$$

in the lowest filling approximation. Roughly speaking the $V_{A}(\vec{r})$ adjusts the single-particle down-spin density,

$$
\left\langle\Phi_{N, N}\left|: \rho_{\downarrow}(\vec{r}):\right| \Phi_{N, N}\right\rangle
$$

while the $N$-body fixed-point constraint insures that the single-particle densities are equal for both spins,

$$
\left\langle\Phi_{N, N}\left|: \rho_{\uparrow}(\vec{r}):\right| \Phi_{N, N}\right\rangle=\left\langle\Phi_{N, N}\left|: \rho_{\downarrow}(\vec{r}):\right| \Phi_{N, N}\right\rangle
$$

Applications of this approach to non-uniform systems will be discussed in a future publication. 


\section{SUMMARY}

We have presented a many-body approach called the symmetric heavy-light ansatz. It is an approximate method for finding the ground state energy of dilute unpolarized twocomponent fermions with attractive interactions. Although the Hamiltonian has an exact $Z_{2}$ symmetry, the ansatz breaks the symmetry by changing the ratio of the masses of the two components. We considered the extreme limit where one component is infinitely heavy and the many-body problem can be solved in terms of single-particle orbitals. The original $Z_{2}$ symmetry was reintroduced by setting the $N$-body density correlations for the two components equal for all $N$. A Markov chain algorithm was designed to generate exactly this $N$-body density constraint.

To test the method we first compared the results of the symmetric heavy-light ansatz with exact Lanczos results for the four-body system in three dimensions. We then tested the method with exact results for four- and six-body systems in both one and two dimensions. We then considered dimer-dimer scattering and larger systems in three dimensions at unitarity and arbitrary values for the scattering length. The results indicate that the method is quite accurate and robust. We discussed some extensions beyond the lowest filling approximation and applications to non-uniform systems.

\section{ACKNOWLEDGEMENTS}

The author thanks Gautam Rupak and Thomas Schäfer for discussions and comments. This work is supported in part by DOE grant DE-FG02-03ER41260.

\section{APPENDIX A: FINITE-VOLUME SCATTERING IN THREE DIMENSIONS}

In this appendix we use Lüscher's formula [31, 32, 33, 34] to relate the coefficient $C$ in the three-dimensional Hubbard model to the S-wave scattering length. We consider one up-spin particle and one down-spin particle in a periodic cube of length $L$. Lüscher's formula relates the two-particle energy levels in the center-of-mass frame to the S-wave phase shift,

$$
p \cot \delta_{0}(p)=\frac{1}{\pi L} S(\eta), \quad \eta=\left(\frac{L p}{2 \pi}\right)^{2}
$$


where $S(\eta)$ is the three-dimensional zeta function,

$$
S(\eta)=\lim _{\Lambda \rightarrow \infty}\left[\sum_{\vec{n}} \frac{\theta\left(\Lambda^{2}-\vec{n}^{2}\right)}{\vec{n}^{2}-\eta}-4 \pi \Lambda\right] .
$$

For $|\eta|<1$ we can expand in powers of $\eta$,

$$
\begin{aligned}
S(\eta) & =-\frac{1}{\eta}+\lim _{\Lambda \rightarrow \infty}\left[\sum_{\vec{n} \neq \overrightarrow{0}} \frac{\theta\left(\Lambda^{2}-\vec{n}^{2}\right)}{\vec{n}^{2}-\eta}-4 \pi \Lambda\right] \\
& =-\frac{1}{\eta}+S_{0}+S_{1} \eta^{1}+S_{2} \eta^{2}+S_{3} \eta^{3} \cdots,
\end{aligned}
$$

where

$$
\begin{gathered}
S_{0}=\lim _{\Lambda \rightarrow \infty}\left[\sum_{\vec{n} \neq \overrightarrow{0}} \frac{\theta\left(\Lambda^{2}-\vec{n}^{2}\right)}{\vec{n}^{2}}-4 \pi \Lambda\right], \\
S_{j}=\sum_{\vec{n} \neq \overrightarrow{0}} \frac{1}{\left(\vec{n}^{2}\right)^{j+1}} \quad j \geq 1 .
\end{gathered}
$$

The first few coefficients are

$$
\begin{aligned}
& S_{0}=-8.913631, \quad S_{1}=16.532288, \quad S_{2}=8.401924, \quad S_{3}=6.945808 \\
& S_{4}=6.426119, \quad S_{5}=6.202149, \quad S_{6}=6.098184, \quad S_{7}=6.048263
\end{aligned}
$$

For small momenta the effective range expansion gives

$$
p \cot \delta_{0}(p) \approx-\frac{1}{a_{\text {scatt }}}+\frac{1}{2} r_{0} p^{2}+\cdots,
$$

where $a_{\text {scatt }}$ is the scattering length and $r_{0}$ is the effective range.

In terms of $\eta$, the energy of the two-body scattering state is

$$
E_{\text {pole }}=\frac{p^{2}}{m}=\frac{\eta}{m}\left(\frac{2 \pi}{L}\right)^{2} .
$$

We compute the two-particle scattering pole at energy $E_{\text {pole }}$ by summing the two-particle bubble diagrams shown in Fig. 13, For spin-dependent masses $m_{\uparrow}$ and $m_{\downarrow}$ the single-particle dispersion relations are

$$
\begin{aligned}
& \omega_{\uparrow}(\vec{p})=\frac{1}{m_{\uparrow}} \sum_{l}\left(1-\cos p_{l}\right), \\
& \omega_{\downarrow}(\vec{p})=\frac{1}{m_{\downarrow}} \sum_{l}\left(1-\cos p_{l}\right) .
\end{aligned}
$$




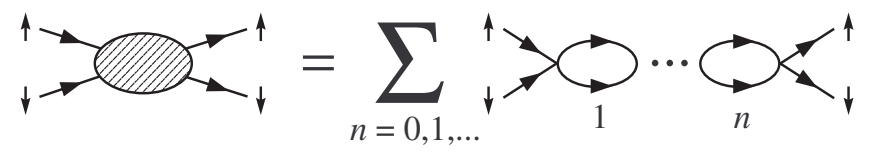

FIG. 13: Sum of bubble diagrams contributing to two-particle scattering

After summing the bubble diagrams as a geometric series, the relation between $C$ and $E_{\text {pole }}$ is

$$
-\frac{1}{C}=\lim _{L \rightarrow \infty} \frac{1}{L^{3}} \sum_{\vec{k} \text { integer }} \frac{1}{-E_{\text {pole }}+\omega_{\uparrow}(2 \pi \vec{k} / L)+\omega_{\downarrow}(2 \pi \vec{k} / L)} .
$$

When combined with Eq. (A1), (A7), and (A8), we can relate $C$ and the scattering length $a_{\text {scatt }}$. We note that for both $H$ and $H_{\mathrm{HL}}$ we have

$$
\omega_{\uparrow}(2 \pi \vec{k} / L)+\omega_{\downarrow}(2 \pi \vec{k} / L)=\frac{2}{m} \sum_{l}\left[1-\cos \left(2 \pi k_{l} / L\right)\right] .
$$

Therefore the coefficient $C$ is exactly the same in both cases. The unitarity limit corresponds with the value $U / t=2 m C=-7.914$.

[1] D. M. Eagles, Phys. Rev. 186, 456 (1969).

[2] A. J. Leggett, in Modern Trends in the Theory of Condensed Matter. Proceedings of the XVIth Karpacz Winter School of Theoretical Physics, Karpacz, Poland, 1980 (SpringerVerlag, Berlin, 1980), p. 13.

[3] P. Nozieres and S. Schmitt-Rink, J. Low Temp. Phys. 59, 195 (1985).

[4] K. M. O'Hara, S. L. Hemmer, M. E. Gehm, S. R. Granade, and J. E. Thomas, Science 298, 2179 (2002).

[5] S. Gupta, Z. Hadzibabic, M. W. Zwierlein, C. A. Stan, K. Dieckmann, C. H. Schunck, E. G. M. van Kempen, B. J. Verhaar, and W. Ketterle, Science 300, 1723 (2003).

[6] C. A. Regal and D. S. Jin, Phys. Rev. Lett. 90, 230404 (2003).

[7] T. Bourdel, J. Cubizolles, L. Khaykovich, K. M. F. Magalhaes, S. J. J. M. F. Kokkelmans, G. V. Shlyapnikov, and C. Salomon, Phys. Rev. Lett. 91, 020402 (2003).

[8] M. E. Gehm, S. L. Hemmer, S. R. Granade, K. M. O’Hara, and J. E. Thomas, Phys. Rev. A68, 011401(R) (2003). 
[9] M. Bartenstein, A. Altmeyer, S. Riedl, S. Jochim, C. Chin, J. Hecker Denschlag, and R. Grimm, Phys. Rev. Lett. 92, 120401 (2004).

[10] J. Kinast, A. Turlapov, J. E. Thomas, Q. Chen, J. Stajic, and K. Levin, Science 307, 1296 (2005), cond-mat/0502087.

[11] O. Penrose and L. Onsager, Phys. Rev. 104, 576 (1956).

[12] L. P. Gor'kov, Soviet Phys. JETP 7, 505 (1958).

[13] D. S. Petrov, Phys. Rev. A67, 010703(R) (2003).

[14] R. d. L. Kronig and W. G. Penney, Proc. Roy. Soc. (London) A130, 499 (1931).

[15] J.-W. Chen and D. B. Kaplan, Phys. Rev. Lett. 92, 257002 (2004), hep-lat/0308016.

[16] D. Lee and T. Schäfer, Phys. Rev. C72, 024006 (2005), nucl-th/0412002.

[17] D. Lee, B. Borasoy, and T. Schäfer, Phys. Rev. C70, 014007 (2004), nucl-th/0402072.

[18] M. Wingate (2005), cond-mat/0502372.

[19] A. Bulgac, J. E. Drut, and P. Magierski, Phys. Rev. Lett. 96, 090404 (2006), condmat/0505374.

[20] D. Lee and T. Schäfer, Phys. Rev. C73, 015201 (2006), nucl-th/0509017.

[21] D. Lee and T. Schäfer, Phys. Rev. C73, 015202 (2006), nucl-th/0509018.

[22] D. Lee, Phys. Rev. B73, 115112 (2006), cond-mat/0511332.

[23] E. Burovski, N. Prokofev, B. Svistunov, and M. Troyer, Phys. Rev. Lett. 96, 160402 (2006), cond-mat/0602224.

[24] E. Burovski, N. Prokofev, B. Svistunov, and M. Troyer, New J. Phys. 8, 153 (2006), condmat/0605350.

[25] D. Lee, Phys. Rev. B75, 134502 (2007), cond-mat/0606706.

[26] D. Lee, Phys. Rev. Lett. 98, 182501 (2007), nucl-th/0701041.

[27] D. Lee and R. Thomson, Phys. Rev. C75, 064003 (2007), nucl-th/0701048.

[28] C. Lanczos, J. Res. Nat. Bur. Stand. 45, 255 (1950).

[29] W. Kohn and L. J. Sham, Phys. Rev. 140, A1133 (1965).

[30] D. Lee, Phys. Rev. A73, 063204 (2006), physics/0512085.

[31] M. Lüscher, Commun. Math. Phys. 105, 153 (1986).

[32] S. R. Beane, P. F. Bedaque, A. Parreno, and M. J. Savage, Phys. Lett. B585, 106 (2004), hep-lat/0312004.

[33] R. Seki and U. van Kolck, Phys. Rev. C73, 044006 (2006), nucl-th/0509094. 
[34] B. Borasoy, E. Epelbaum, H. Krebs, D. Lee, and U.-G. Meißner, Eur. Phys. J. A31, 105 (2007), nucl-th/0611087.

[35] G. Rupak (2006), nucl-th/0605074.

[36] D. S. Petrov, C. Salomon, and G. V. Shlyapnikov, Phys. Rev. Lett. 93, 090404 (2004).

[37] J. Carlson, S. Y. Chang, V. R. Pandharipande, and K. Schmidt, Phys. Rev. Lett. 91, 50401 (2003), physics/0303094.

[38] S. Y. Chang, V. R. Pandharipande, J. Carlson, and K. E. Schmidt, Phys. Rev. A70, 043602 (2004).

[39] G. E. Astrakharchik, J. Boronat, J. Casulleras, and S. Giorgini, Phys. Rev. Lett. 93, 200404 (2004), cond-mat/0406113.

[40] J. R. Engelbrecht, M. Randeria, and C. S. de Melo, Phys. Rev. B55, 15153 (1997).

[41] G. A. Baker, Phys. Rev. C60, 054311 (1999).

[42] H. Heiselberg, Phys. Rev. A 63, 043606 (2001), cond-mat/0002056.

[43] A. Perali, P. Pieri, and G. C. Strinati, Phys. Rev. Lett. 93, 100404 (2004).

[44] T. Papenbrock, Phys. Rev. A 72, 041603 (2005), cond-mat/0507183.

[45] T. Schäfer, C.-W. Kao, and S. R. Cotanch, Nucl. Phys. A762, 82 (2005), nucl-th/0504088.

[46] J. Chen, Chinese Phys. Lett 24, 1825 (2007), nucl-th/0602065.

[47] Y. Nishida and D. T. Son, Phys. Rev. Lett. 97, 050403 (2006), cond-mat/0604500.

[48] Y. Nishida and D. T. Son, Phys. Rev. A 75, 063617 (2007), cond-mat/0607835.

[49] M. Y. Veillette, D. E. Sheehy, and L. Radzihovsky, Phys. Rev. A 75, 043614 (2007), condmat/0610798.

[50] P. Arnold, J. E. Drut, and D. T. Son, Phys. Rev. A 75, 043605 (2007), cond-mat/0608477.

[51] O. Juillet, New Journal of Physics 9, 163 (2007), cond-mat/0609063.

[52] T. Abe and R. Seki (2007), arXiv:0708.2524 [nucl-th].

[53] A. Bulgac, J. E. Drut, P. Magierski, and G. Wlazlowski (2008), arXiv.org:0801.1504.

[54] J. T. Stewart, J. P. Gaebler, C. A. Regal, and D. S. Jin, Phys. Rev. Lett. 97, 220406 (2006), arXiv.org:cond-mat/0607776.

[55] K. Huang and C. N. Yang, Phys. Rev. 105, 767 (1957).

[56] T. D. Lee and C. N. Yang, Phys. Rev. 105, 1119 (1957).

[57] J.-W. Chen and E. Nakano, Phys. Rev. A75, 043620 (2007), cond-mat/0610011.

[58] T. Papenbrock and A. Bhattacharyya, Phys. Rev. C75, 014304 (2007), nucl-th/0609084. 
[59] J. Engel, Phys. Rev. C75, 014306 (2007), nucl-th/0610043.

[60] R. J. Furnstahl (2007), nucl-th/0702040.

[61] A. Bulgac, Phys. Rev. A76, 040502(R) (2007), cond-mat/0703526.

[62] R. J. Furnstahl, G. Rupak, and T. Schafer (2008), arXiv:0801.0729 [nucl-th]. 